\title{
Multiple-Resampling Receiver Design for OFDM Over Doppler-Distorted Underwater Acoustic Channels
}

\author{
Kai Tu, Tolga M. Duman, Fellow, IEEE, Milica Stojanovic, Fellow, IEEE, and John G. Proakis, Life Fellow, IEEE
}

\begin{abstract}
In this paper, we focus on orthogonal frequency-division multiplexing (OFDM) receiver designs for underwater acoustic (UWA) channels with user- and/or path-specific Doppler scaling distortions. The scenario is motivated by the cooperative communications framework, where distributed transmitter/receiver pairs may experience significantly different Doppler distortions, as well as by the single-user scenarios, where distinct Doppler scaling factors may exist among different propagation paths. The conventional approach of front-end resampling that corrects for common Doppler scaling may not be appropriate in such scenarios, rendering a post-fast-Fourier-transform (FFT) signal that is contaminated by user- and/or path-specific intercarrier interference. To counteract this problem, we propose a family of front-end receiver structures that utilize multiple-resampling(MR)branches, each matched to the Doppler scaling factor of a particular user and/or path. Following resampling, FFT modules transform the Doppler-compensated signals into the frequency domain for further processing through linear or nonlinear detection schemes. As part of the overall receiver structure, a gradient-descent approach is also proposed to refine the channel estimates obtained by standard sparse channel estimators. The effectiveness and robustness of the proposed receivers are demonstrated via simulations, as well as emulations based on real data collected during the 2010 Mobile Acoustic Communications Experiment (MACE10, Martha's Vineyard, MA) and the 2008 Kauai Acomms MURI (KAM08, Kauai, HI) experiment.
\end{abstract}

Index Terms - Channel estimation, Doppler effect, interference suppression, matched filters, multiple-input-multiple-output (MIMO), multiuser detection, orthogonal frequency-division multiplexing (OFDM), underwater communication, time-varying channels.

\section{INTRODUCTION}

A S demonstrated in several recent shallow-water acoustic communications experiments, orthogonal frequency-division multiplexing (OFDM) in both single-input-single-output

Manuscript received June 09, 2011; revised March 28, 2012; accepted September 25, 2012. Date of publication December 21, 2012; date of current version April 10, 2013. This work was supported by the Multidisciplinary University Research Initiative (MURI) Office of Naval Research (ONR) under Grants N00014-07-1-0739/0738, N00014-10-1-0576, and N00014-09-1-0700.

Associate Editor: J. Gomes.

$\mathrm{K}$. Tu was with the School of Electrical, Computer and Energy Engineering, Arizona State University, Tempe, AZ 85287-5706 USA. He is now with Qualcomm Inc., San Diego, CA 92121 USA (e-mail: kai.tu@asu.edu).

T. M. Duman is with the Department of Electrical and Electronics Engineering, Bilkent University, Bilkent, Ankara TR-06800, Turkey, on leave from the School of Electrical, Computer and Energy Engineering, Arizona State University, Tempe, AZ 85287-5706 USA.

M. Stojanovic is with the Department of Electrical and Computer Engineering, Northeastern University, Boston, MA 02115 USA.

J. G. Proakis is with the Department of Electrical and Computer Engineering, University of California San Diego, La Jolla, CA 92093 USA.

Color versions of one or more of the figures in this paper are available online at http://ieeexplore.ieee.org.

Digital Object Identifier 10.1109/JOE.2012.2221812
(SISO) and multiple-input-multiple-output (MIMO) configurations is capable of dealing with the large multipath spreads of underwater acoustic (UWA) channels without resorting to complicated equalizer structures essential for the case of single-carrier systems [1]-[4]. While acoustic OFDM systems have mostly been tested for single-user (point-to-point) transmissions, it is also possible to deploy them in a multiuser environment. Cooperative multiuser systems, differently from their single-user counterparts where both the transmitting and receiving elements are colocated, are formed by geographically separated transmitting and/or receiving elements [5]. These systems leverage multiple users as an extra degree of freedom and promise large gains for both capacity [6] and reliability [7]. While multiuser MIMO may include a broad range of configurations, such as MIMO broadcast [8], MIMO multiple access (MAC) [9], and network MIMO [10], in this paper, the focus is on MAC channels, where distributed users, operating simultaneously in the same frequency band, transmit independent data streams to a centralized receiver with colocated receiving elements. The major challenge for both single-user and multiuser systems is that with the low propagation speed of sound in water (nominally $1500 \mathrm{~m} / \mathrm{s}$ ), the Doppler distortion becomes much more severe than that typically observed in terrestrial radio communications, causing significant time-variation and intercarrier interference (ICI).

To address the issues related to time-variation and Dopplerinduced ICI, a variety of receiver designs have been proposed in the literature [11]-[14]. Although receivers with general ICImitigation techniques are available, such as the adaptive frequency-domain equalizers proposed in [2], most existing receiver designs are dedicated to channels where the only source of ICI is the motion-induced Doppler scaling, i.e., the time compression/dilation that the signal experiences during propagation [13], [15], [16]. In this case, it is typically assumed that all the signal arrivals are characterized by approximately the same Doppler scaling factor, so that resampling the received signal suffices to compensate for the time compression/dilation [15]. While this assumption may be accurate for certain cases, it does not hold in general. Particularly, in multiuser systems, Doppler scaling factors may be very different for different users, since users are likely to move in different directions with respect to the receiver, as illustrated in Fig. 1. Here, node 0 moves perpendicularly with respect to the receiver, rendering a Doppler scaling factor $a_{0} \approx 0$, while nodes 1 and 2 move toward and away from the receiver, respectively, giving rise to Doppler scaling factors $a_{1}>0$ and $a_{2}<0$. Note also that this disparate nature of Doppler scaling factors may also manifest itself in single-user 


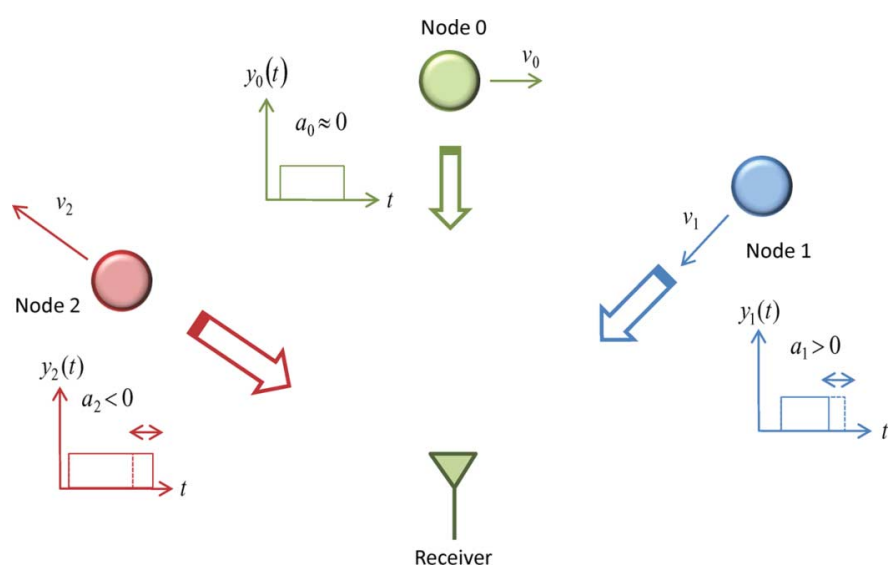

Fig. 1. User-specific Doppler distortions arise from motion.

environments, i.e., given a particular geometry of the propagation paths and the transmitter/receiver motion, significant difference of the Doppler scaling factors may arise when different propagation paths experience significantly different length variations [17].

To address the challenges posed by the disparate Doppler scaling factors, a remedy for the single-resampling (SR) designs has been proposed in [18]. The key idea of this approach is to optimize the resampling rate in such a way that the Fisher information of the resulting equivalent discrete channel model is maximized. To achieve this goal, Yerramalli and Mitra [18] consider two different optimization criteria: 1) maximization of the trace of the Fisher information matrix; and 2) minimization of the mean squared channel estimation error. While conceptually appealing, due to the complexity of the cost function, the closed-form expression for the optimal resampling rate is intractable (particularly when the first criterion is applied), and therefore, a brute-force approach may be needed. Applying the second criterion, the authors obtain a suboptimal solution, which indicates that when the received signal is dominated by one strong arrival, the resampling rate should be equal to the Doppler scaling factor of that arrival, while when the arrivals have almost equal power, the resampling rate should be the average of their associated Doppler scaling factors. We point out that while properly choosing the resampling rate may improve the performance, in situations where different arrivals have markedly different Doppler scales, the residual Doppler distortion will still be significant, causing severe user- and/or path-specific ICI that degrades the system performance.

In this paper, we address the design of a receiver structure that copes with the challenges posed by user- and/or path-specific Doppler distortions. We find that the receiver should include multiple-resampling (MR) branches, one for each distinct Doppler scaling factor associated with different users or different propagation paths of the same user. Fast Fourier transform (FFT) demodulation of the resampling branch outputs yields a set of Doppler-compensated frequency-domain samples, which can subsequently be exploited by various detection schemes. In particular, for the user-specific Doppler case, two custom designs are introduced, namely: 1) a regularized linear detector that deals with the redundancy in the observation vector; and 2) a new nonlinear detector that seeks additional performance gains through interference cancellation (IC). For the path-specific Doppler case, linear detection based on minimum mean square error (MMSE) optimization is adopted. For all of these schemes, we explicitly estimate the path gains, delays, and Doppler scaling factors of the pertinent arrivals. Particularly, we propose a robust two-step sparse channel estimation approach which exploits the initial channel estimates obtained by standard techniques [16], [17] and provides a gradient-descent-based refinement step to cope with the basis mismatch problem. We demonstrate the effectiveness of these receivers both through simulations and using real data recorded during the 2010 Mobile Acoustic Communications Experiment (MACE10, Martha's Vineyard, MA) [19] and the 2008 Kauai Acomms MURI (KAM08, Kauai, HI) experiment [20]. In particular, to emulate a data set with an extensive set of Doppler rate differences for different users, we resample the recorded signal at different rates and add the resampled signals to form a compound signal, which is used as an input to different receiver structures. To assess the performance of the proposed receivers, we compare the results with those obtained by a standard receiver, for which only an SR branch is employed.

The paper is organized as follows. Section II contains the OFDM system description. Section III focuses on receiver designs for the user-specific Doppler case. We derive the front-end receiver structure based on an efficient FFT implementation. Custom-designed detection schemes are also discussed. In Section IV, we continue on discussions of the path-specific Doppler case. Section V presents some practical considerations involving sparse channel estimation schemes. Section VI offers simulation results and experimental data studies, and finally, Section VII concludes the paper.

\section{System Model AND Preliminaries}

In this section, we establish mathematical models for multiuser and single-user UWA OFDM transmission scenarios. For the former, our focus is the user-specific Doppler distortion, for which we use a distributed MIMO system model (i.e., a $\mathrm{MAC}$ ), while for the latter, the path-specific Doppler distortion is studied.

\section{A. User-Specific Doppler}

Consider an $N_{t}$-user, $N$-subcarrier OFDM system with a cyclic prefix (CP) of duration $T_{g}$, a block duration $T$, and modulation symbols belonging to a complex-valued constellation. The transmitted signal of the $i$ th user can be written as [21]

$$
s_{i}(t)=\operatorname{Re}\left\{\sum_{k=0}^{N-1} d_{k}^{(i)} e^{j 2 \pi f_{k} t} R(t)\right\}
$$

where $\left\{d_{k}^{(i)}\right\}$ are the data symbols modulated onto the frequency $f_{k}=f_{0}+k / T$ of the $i$ th user, $R(t)$ is a rectangular pulse of duration $T+T_{g},{ }^{1}$ and $\operatorname{Re}\{\cdot\}$ denotes the real part. We consider a scenario where a centralized receiver, equipped with $N_{r}$ receiving elements, simultaneously receives $N_{t}$ independent data streams $\mathbf{d}^{(1)}, \ldots, \mathbf{d}^{\left(N_{t}\right)}$ from $N_{t}$ geographically separated

\footnotetext{
${ }^{1}$ Results in this paper can be extended to nonrectangular pulses as long as the pulses are sufficiently smooth.
} 


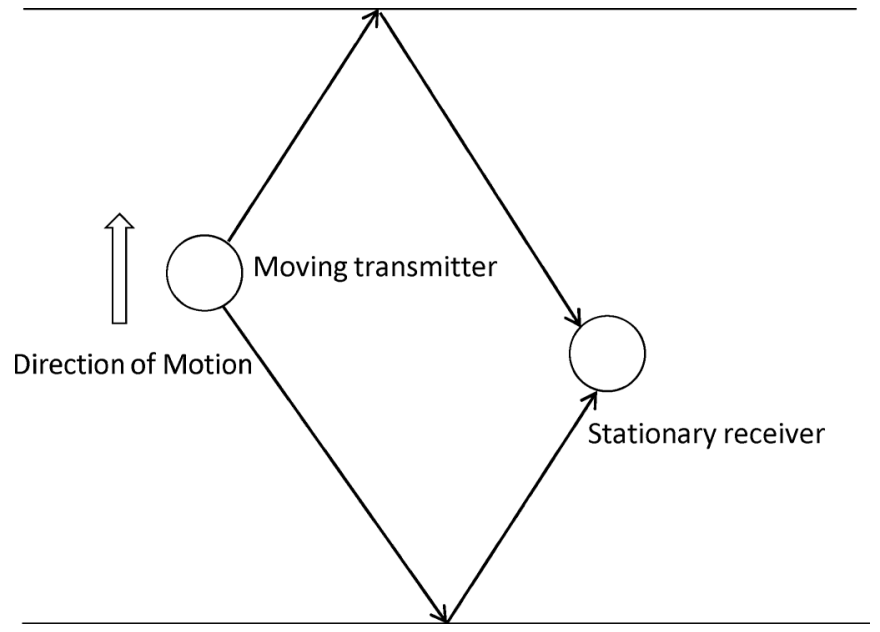

Fig. 2. Path-specific Doppler can arise due to motion.

transmitters, where $\mathbf{d}^{(i)}=\left[d_{0}^{(i)}, \ldots, d_{N-1}^{(i)}\right]^{\mathrm{T}}$. Throughout this paper, we assume that the $\mathrm{CP}$ is sufficiently long to prevent interblock interference, ${ }^{2}$ and we focus on a single OFDM block. Further, we assume that the user transmissions are accurately synchronized, so that their relative delays at the receiver are less than the duration of the cyclic prefix. This model is mathematically the same as that of a centralized MIMO system employing spatial multiplexing [22], but the practical distinction is that the Doppler factors can be substantially different with distributed transmitters.

We start by considering a UWA channel, where, for each transmitter/receiver pair $(i, m), N_{p}^{(i, m)}$ propagation paths are present, and, for simplicity, all the paths corresponding to a particular user are assumed to share a common Doppler scaling factor. ${ }^{3}$ The input-output relationship in the absence of noise can be expressed as

$$
y_{m}(t)=\sum_{i=1}^{N_{t}} \sum_{p=0}^{N_{p}^{(i, m)}-1} h_{p}^{(i, m)} s_{i}\left(t+a^{(i)} t-\tau_{p}^{(i, m)}\right)
$$

where $h_{p}^{(i, m)}$ and $\tau_{p}^{(i, m)}$ are, respectively, the path gain and delay of the $p$ th path, and $a^{(i)}$ is the Doppler scaling factor associated with the $i$ th user. Denoting by $v^{(i)}$ the relative transmitter/receiver velocity, the user-specific Doppler scaling factor is $a^{(i)}=v^{(i)} / v_{s}$, where $v_{s}$ is the speed of sound in water. For typical UWA systems, we have that $\left|a^{(i)}\right| \ll 1$, but possibly on the order of $10^{-3}$ or $10^{-4}$. Note that in a mobile multiuser scenario, each user's signal is likely to be dominated by a single Doppler factor, in which case, model (2) applies approximately. Note also that the channel is time varying; in many cases, parameters $h_{p}^{(i, m)}, \tau_{p}^{(i, m)}$, and $a^{(i)}$ change slowly and can be taken as constants for the frame duration.

\footnotetext{
${ }^{2}$ The assumption is practical only when the channel's impulse response is small with respect to the OFDM symbol length, in which case the existence of a CP long enough to handle the channel would not cause significant decrease in spectral efficiency.

${ }^{3}$ While, in general, the Doppler scaling that arises in multiuser systems can be both user specific and path specific, for simplicity of illustration, we only consider cases affected by user-specific Doppler scaling and defer the path-specific cases to Section II-B.
}

Based on (2), we can write the received (bandpass) signal as

$$
\begin{array}{r}
\widetilde{v}_{m}(t)=\operatorname{Re}\left\{\sum_{i=0}^{N_{t}} \sum_{k=0}^{N-1} \sum_{p=0}^{N_{p}^{(i, m)}-1} d_{k}^{(i)} h_{p}^{(i, m)} e^{j 2 \pi f_{k}\left(t+a^{(i)} t-\tau_{p}^{(i, m)}\right)}\right. \\
\left.\quad \times R\left(t+a^{(i)} t-\tau_{p}^{i, m}\right)\right\}+\widetilde{w}_{m}(t)
\end{array}
$$

where $\widetilde{w}_{m}(t)$ is additive white Gaussian noise (AWGN), independent between receiving elements, with power spectral density (PSD) $N_{0} / 2 .{ }^{4}$ Equivalently, adopting the complex envelope representation with respect to frequency $f_{0}$, the model of the received signal yields

$$
v_{m}(t)=\sum_{i=1}^{N_{t}} \sum_{k=0}^{N-1} d_{k}^{(i)} P_{k}^{(i, m)}(t)+w_{m}(t)
$$

where $w_{m}(t)$ is a circularly symmetric complex AWGN with PSD $N_{0}$ per complex dimension, and

$$
\begin{aligned}
P_{k}^{(i, m)}(t)= & \sum_{p=0}^{N_{p}^{(i, m)}-1} \alpha_{p}^{(i, m)}(k) e^{j 2 \pi a^{(i)} f_{0} t} e^{\frac{j 2 \pi\left(t+a^{(i)} t\right) k}{T}} \\
& \times R\left(t+a^{(i)} t-\tau_{p}^{(i, m)}\right) \\
\alpha_{p}^{(i, m)}(k)= & h_{p}^{(i, m)} e^{-j 2 \pi f_{k} \tau_{p}^{(i, m)}} .
\end{aligned}
$$

\section{B. Path-Specific Doppler}

To illustrate the path-specific Doppler distortion, we consider a single-user SISO system - extensions to MIMO systems are obvious. Grouping the propagation paths into $N_{c}$ clusters, we assume that each cluster is associated with a distinct Doppler scaling factor. For example, as illustrated in Fig. 2, this model may apply to a scenario where the surface- and bottom-reflected paths are associated with significantly different angles of arrival and the transmitter movement is toward the ocean surface.

Following a similar procedure as in Section II-A, we express the baseband signal of the receiving element as

$$
v(t)=\sum_{k=0}^{N-1} d_{k} P_{k}(t)+w(t)
$$

where $d_{k}$ is the symbol transmitted at the $k$ th subcarrier, $w(t)$ is a circularly symmetric complex AWGN with PSD $N_{0}$ per complex dimension, and

$$
\begin{aligned}
P_{k}(t)= & \sum_{c=0}^{N_{c}-1} \sum_{p=0}^{N_{p}^{(c)}-1} \alpha_{p}^{(c)}(k) e^{j 2 \pi a_{c} f_{0} t} e^{j 2 \pi\left(t+a_{c} t\right) k / T} \\
& \times R\left(t+a_{c} t-\tau_{c, p}\right) \\
\alpha_{p}^{(c)}(k)= & h_{c, p} e^{-j 2 \pi f_{k} \tau_{c, p}} .
\end{aligned}
$$

Here, $N_{p}^{(c)}$ is the number of paths in the $c$ th cluster; and $h_{c, p}$, $\tau_{c, p}$, and $a_{c}$ are, respectively, the path gain, delay, and Doppler scaling factor of the $p$ th path in the $c$ th cluster.

${ }^{4}$ Noise in a UWA channel is, in general, colored, but we focus for simplicity on the white noise case as an illustrative example. Extensions of the results to a specific noise PSD are straightforward, as will become clear later. 


\section{Multiuser Receiver Design With USER-SPECIFIC DOPPLER}

Consider multiple geographically separated users transmitting independent data streams to a centralized receiver. Users from different locations operate in the same frequency band, and multiuser detection is made possible by combining the signals received at multiple receiving elements to exploit the spatial diversity. In this section, we consider user-specific Doppler distortion; path-specific scenarios will be discussed in Section IV.

\section{A. MR-Based Receiver Front-End}

We consider transmission of a single OFDM block assuming perfect channel state information (CSI) at the receiver, i.e., knowledge of Doppler scaling factors as well as channel path gains and delays. Since the noise is white Gaussian and independent between receiving elements, maximum-likelihood (ML) data detection aims to find the sequence $\mathbf{d}=\left[\mathbf{d}^{(1)^{\mathrm{T}}}, \ldots, \mathbf{d}^{\left(N_{t}\right)^{\mathrm{T}}}\right]^{\mathrm{T}}$, which minimizes the metric

$$
\begin{aligned}
& \Lambda_{m}(\mathbf{d})=\int_{-\infty}^{\infty}\left|v(t)-\sum_{i=1}^{N_{t}} \sum_{k=0}^{N-1} d_{k}^{(i)} P_{k}^{(i, m)}(t)\right|^{2} d t \\
& \forall m \in\left\{1, \ldots, N_{r}\right\} .
\end{aligned}
$$

This metric implies a set of frequency-domain samples given by

$$
y_{k}^{(i, m)}=\int_{-\infty}^{\infty} v_{m}(t) P_{k}^{(i, m)^{*}}(t) d t, \quad k=0,1, \ldots, N-1 .
$$

Since the number of subcarriers is typically large, direct implementation of $N$ parallel matched-filter branches is clearly not a viable option. Thus, we focus on an alternative interpretation of (9), namely, we first restrict our attention to the time interval that contains the signal but not its cyclic extension, ${ }^{5}$ which effectively yields

$$
\begin{aligned}
y_{k}^{(i, m)} \approx & \int_{0}^{\frac{T}{1+a^{(i)}}} v_{m}(t) \sum_{p=0}^{N_{p}^{(i, m)}-1} \alpha_{p}^{(i, m)}(k)^{*} e^{-j 2 \pi a^{(i)} f_{0} t} \\
= & \int_{0}^{\frac{T}{1+a^{(i)}}} \underbrace{v_{m}(t) e^{-j 2 \pi\left(t+a^{(i)} t\right) k / T} d t}_{v_{m}^{(i)}(t)} \sum_{p=0}^{\left(^{(i)} f_{0} t\right.} \alpha_{p}^{(i, m)}(k)^{*} \\
& \times e^{-j 2 \pi\left(t+a^{(i)} t\right) k / T} d t
\end{aligned}
$$

where $v_{m}^{(i)}(t)$ is obtained by compensating for the Doppler-induced frequency shift $a^{(i)} f_{0}$. Equivalently, we can write

$$
y_{k}^{(i, m)} \approx \alpha^{(i, m)}(k)^{*} \tilde{y}_{k}^{(i, m)}
$$

${ }^{5}$ While for Doppler-affected channels, the signal in the guard interval (in our case, the cyclic extension) may contain nonredundant information [23], for simplicity, we follow the standard approach to consider only the part excluding the cyclic extension for data detection [21]. with

$$
\alpha^{(i, m)}(k)=\sum_{p=0}^{N_{p}^{(i, m)}-1} \frac{\alpha_{p}^{(i, m)}(k)}{1+a^{(i)}}
$$

and

$$
\tilde{y}_{k}^{(i, m)}=\left(1+a^{(i)}\right) \int_{0}^{\frac{T}{1+a^{(i)}}} v_{m}^{(i)}(t) e^{-j 2 \pi\left(1+a^{(i)}\right) k t / T} d t .
$$

This computation can be efficiently carried out for all the subcarriers by a single FFT. Specifically, introducing a change of variable $\xi=\left(1+a^{(i)}\right) t$ in (12) results in

$$
\widetilde{y}_{k}^{(i, m)}=\int_{0}^{T} v_{m}^{(i)}\left(\frac{\xi}{1+a^{(i)}}\right) e^{-j 2 \pi k \xi / T} d \xi
$$

where the integration, when carried out in the discrete-time domain, is just an FFT. From (13), we also notice that evaluation of $\tilde{y}_{k}^{(i, m)}(t)$ requires resampling of $v_{m}^{(i)}(t)$ according to the Doppler scaling factor $a^{(i)}$ when there are $N_{t}$ distinct Doppler scaling factors. In other words, one needs to resample the received signal $v_{m}(t) N_{t}$ times, each time according to a distinct Doppler scaling factor.

Fig. 3 shows the block diagram of the resulting receiver front-end. It consists of $N_{t}$ parallel branches, each one associated with the Doppler scaling factor of a particular user. Each branch performs frequency shift, resampling, and FFT. The implementation complexity thus increases only linearly with the number of distinct Doppler scaling factors, and the processing can be performed in parallel, rendering a computationally affordable solution.

\section{B. Data Detection}

To design a detection algorithm, it is helpful to define an equivalent discrete channel model that relates the acquired samples (10) to the data symbols. Substituting the relations (4) and (5) into the expression (10), we obtain

$$
y_{k}^{(i, m)}=\sum_{u=1}^{N_{t}} \sum_{l=0}^{N-1} \Phi_{k, l}^{(i, u)}(m) d_{l}^{(i)}+w_{k}^{(i, m)}
$$

where

$$
\Phi_{k, l}^{(i, u)}(m)=\int_{0}^{T} P_{l}^{(u, m)}(t) P_{k}^{(i, m)^{*}}(t) d t
$$

and $w_{k}^{(i, m)}$ is additive Gaussian noise with autocorrelation [24]

$$
\Omega_{k, l}^{(i, u)}(m)=E\left[w_{k}^{(i, m)} w_{l}^{(u, m)^{*}}\right] \approx 2 N_{0} T \cdot \Phi_{k, l}^{(i, u)}(m) .
$$

Grouping all the carriers together, the above expressions can also be represented in a compact form as

$$
\mathbf{y}(i, m)=\boldsymbol{\Phi}(i, m) \mathbf{d}+\mathbf{w}(i, m)
$$




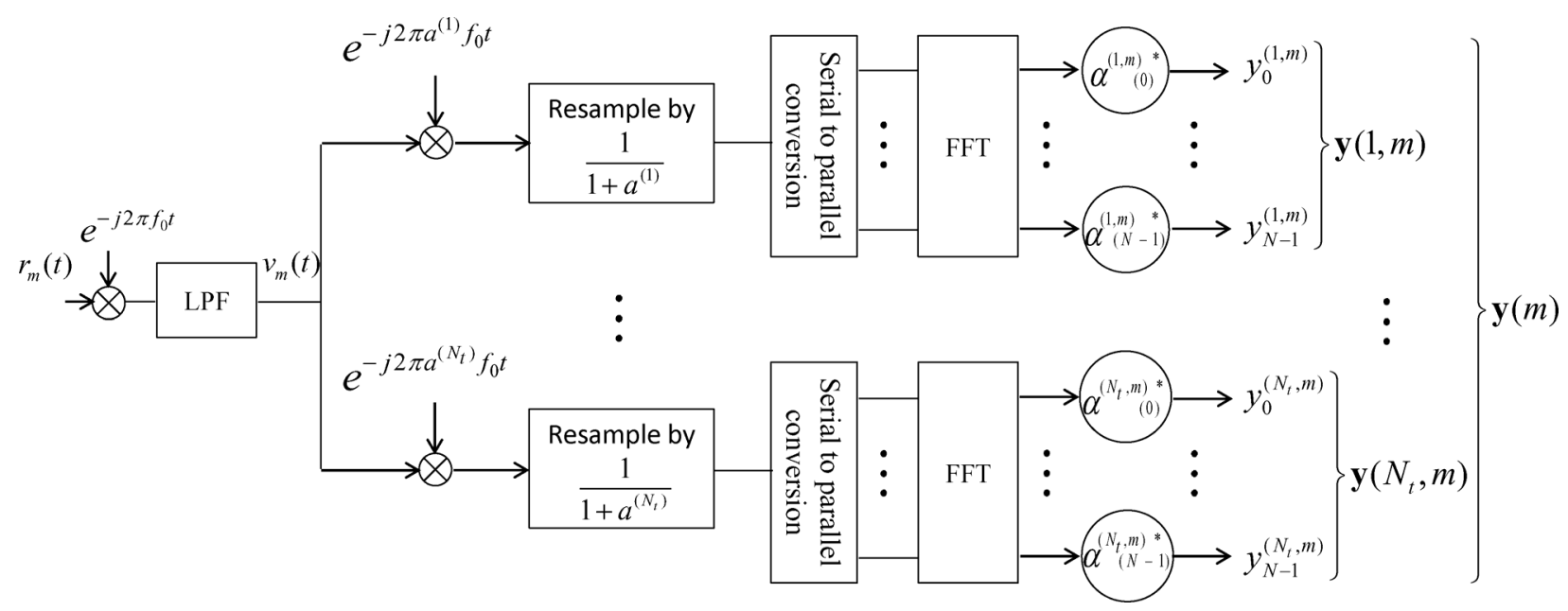

Fig. 3. MR receiver front-end design of the $m$ th receiving element for multiuser MIMO OFDM systems affected by user-specific Doppler distortions.

where

$$
\begin{aligned}
\boldsymbol{\Phi}(i, m) & =\left[\boldsymbol{\Phi}^{(i, 1)}(m), \ldots, \boldsymbol{\Phi}^{\left(i, N_{t}\right)}(m)\right] \\
{\left[\boldsymbol{\Phi}^{(i, u)}(m)\right]_{(k, l)} } & =\Phi_{k, l}^{(i, u)}(m) \\
\mathbf{y}(i, m) & =\left[y_{0}^{(i, m)}, \ldots, y_{N-1}^{(i, m)}\right]^{\mathrm{T}} \\
\mathbf{w}(i, m) & =\left[w_{0}^{(i, m)}, \ldots, w_{N-1}^{(i, m)}\right]^{\mathrm{T}} .
\end{aligned}
$$

The vectors $\mathbf{y}(i, m)$ can now be grouped for all the transmitter/ receiver pairs to obtain the overall MIMO system model

$$
\underbrace{\left[\begin{array}{c}
\mathbf{y}(1,1) \\
\mathbf{y}(2,1) \\
\vdots \\
\mathbf{y}\left(N_{t}, N_{r}\right)
\end{array}\right]}_{\mathbf{y}}=\underbrace{\left[\begin{array}{c}
\boldsymbol{\Phi}(1,1) \\
\mathbf{\Phi}(2,1) \\
\vdots \\
\mathbf{\Phi}\left(N_{t}, N_{r}\right)
\end{array}\right]}_{\mathbf{\Phi}} \mathbf{d}+\underbrace{\left[\begin{array}{c}
\mathbf{w}(1,1) \\
\mathbf{w}(2,1) \\
\vdots \\
\mathbf{w}\left(N_{t}, N_{r}\right)
\end{array}\right]}_{\mathbf{w}} .
$$

The aggregate noise vector $\mathbf{w}$ is characterized by the covariance matrix

$$
\boldsymbol{\Omega}=\left[\begin{array}{cccc}
\boldsymbol{\Omega}(1) & \mathbf{0} & \ldots & \mathbf{0} \\
\mathbf{0} & \boldsymbol{\Omega}(2) & \ldots & \mathbf{0} \\
\mathbf{0} & \mathbf{0} & \ddots & \mathbf{0} \\
\mathbf{0} & \mathbf{0} & \ldots & \boldsymbol{\Omega}\left(N_{r}\right)
\end{array}\right]
$$

with

$$
\boldsymbol{\Omega}(m)=\left[\begin{array}{ccc}
\boldsymbol{\Omega}^{(1,1)}(m) & \ldots & \boldsymbol{\Omega}^{\left(1, N_{t}\right)}(m) \\
\vdots & \ddots & \vdots \\
\boldsymbol{\Omega}^{\left(N_{t}, 1\right)}(m) & \ldots & \boldsymbol{\Omega}^{\left(N_{t}, N_{t}\right)}(m)
\end{array}\right]
$$

whose entries are defined by (16).

1) Linear Detectors: Given (18), we can obtain the commonly used linear MMSE detector. While conceptually simple, the implementation of this detector requires some care since the matrices $\boldsymbol{\Phi}$ and $\boldsymbol{\Omega}$ are both singular. The singularity problem is a direct consequence of the fact that different subsets of the signal vector $\mathbf{y}$ are generated from the same input signal sampled multiple times. As a result, strong dependence exists among different subsets of the frequency-domain samples in different branches, and loss of rank for both $\mathbf{H}$ and $\boldsymbol{\Phi}$ is expected.

The optimal solution can nonetheless be obtained through singular value decomposition (SVD), which can be used to remove the redundant coordinates in $\mathbf{y}$ [25]. Alternatively, a scaled identity matrix can be added before inversion. The resulting regularized linear detector is defined by

$$
\tilde{\mathbf{d}}_{\mathrm{MMSE}}=\operatorname{Dec}\left(\boldsymbol{\Phi}^{\mathrm{H}}\left(\boldsymbol{\Phi} \boldsymbol{\Phi}^{\mathrm{H}}+\boldsymbol{\Omega}+\epsilon \mathbf{I}\right)^{-1} \mathbf{y}\right)
$$

where $\operatorname{Dec}(\cdot)$ represents the symbol decision. For instance, in binary phase-shift keying (BPSK), the symbol decision is taken as the sign of the real part of the symbol estimate. Here, the regularization factor $\epsilon$ is chosen as a small number with respect to the average of the nonzero eigenvalues of $\boldsymbol{\Phi} \boldsymbol{\Phi}^{\mathrm{H}}$. For demonstration purposes, the regularized MMSE detector utilizes the standard matrix-inversion-based implementation. The size of the inverted matrix is $N_{u} N \times N_{u} N$. Interested readers are referred to [26] for approaches exploring possible computational cost reduction techniques.

2) Interference Cancellation: IC [27] is considered as a means of improving the error rate performance of the system. An IC detector forms an estimate of the interference caused by one transmitter to the other, and subtracts this estimate from the desired signal before making symbol decisions. The estimation/detection process is performed iteratively, such that the $n$th iteration, carried out for detection of the first user's data stream, yields an interference estimate

$$
\mathbf{I}_{n}(1, m)=\sum_{i=2}^{N_{t}} \boldsymbol{\Phi}^{(1, i)}(m) \tilde{\mathbf{d}}_{I C}^{(i)}(n-1)
$$

which is used to form the symbol decisions as

$$
\tilde{\mathbf{d}}_{I C}^{(1)}(n)=\operatorname{Dec}\left(\sum_{m=1}^{N_{r}}\left(\mathbf{y}(1, m)-\mathbf{I}_{n}(1, m)\right)\right) .
$$

The process is analogous for the other transmitters. The IC detector is initialized by symbol decisions that can be obtained using one of the linear detectors discussed previously. Note that after resampling, there is no self-ICI since we only consider Doppler shifts, and the only interference is due to the presence 


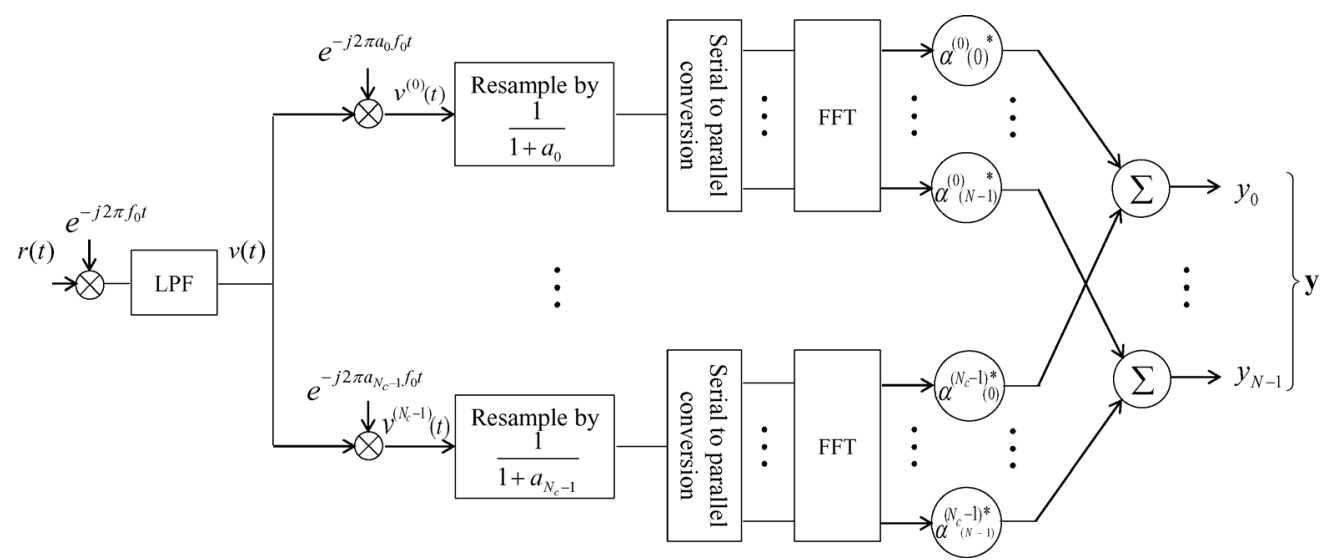

Fig. 4. MR receiver front-end design for single-user OFDM systems affected by path-specific Doppler distortions.

of other users. As will be illustrated through numerical examples, iterative IC detection offers a significant performance improvement over linear detection, while maintaining a relatively low complexity.

\section{Single-User Receiver Design With Path-Specific DOPPLER SCALING}

We now consider receiver designs for the path-specific Doppler scenarios. For ease of illustration, a single-user SISO system is adopted - more general scenarios can be worked out in a straightforward manner [24].

\section{A. MR-Based Receiver Front-End}

Similarly as in Section III, we consider transmission of a single OFDM block assuming perfect receiver CSI. A procedure analogous to that of Section III-A yields an MR front-end in the context of path-specific Doppler by simplifying

$$
y_{k}=\int_{-\infty}^{\infty} v(t) P_{k}^{*}(t) d t, \quad k=0,1, \ldots, N-1
$$

where $y_{k}$ is the matched-filter output, obtained by correlating the received signal $v(t)$ with the equivalent modulation pulse $P_{k}(t)$. With a change of variable $\xi=\left(1+a_{c}\right) t$, expression (21) reduces to

$$
y_{k} \approx \sum_{c=0}^{N_{c}-1} \alpha^{(c)}(k)^{*} \tilde{y}_{k}^{(c)}
$$

where

$$
\alpha^{(c)}(k)=\sum_{p=0}^{N_{p}^{(c)}-1} \frac{\alpha_{p}^{(c)}(k)}{\left(1+a_{c}\right)}
$$

and

$$
\tilde{y}_{k}^{(c)}=\int_{0}^{T} v^{(c)}\left(\frac{\xi}{1+a_{c}}\right) e^{-j 2 \pi k \xi / T} d \xi .
$$

Here, $v^{(c)}(t)=v(t) e^{-j 2 \pi a_{c} f_{0} t}$ is a frequency-shifted version of $v(t)$. According to (22), the received signal $v^{(c)}(t)$ should first be shifted in frequency and resampled to obtain the signals $\left\{v^{(c)}\left(t /\left(1+a_{c}\right)\right)\right\}_{c=0}^{N_{c}-1}$. These signals, when cast in the discrete-time framework, can now be demodulated efficiently using the FFT. Equations (22), (23), and (24) thus define an MR receiver for the path-specific Doppler scenarios, where each branch is associated with one cluster, the output of which are scaled and added together to yield the final demodulated samples. The block diagram of the receiver is shown in Fig. 4.

\section{B. Data Detection}

Similarly as in Section III-B, we first write the discrete channel model that characterizes (21) as

$$
y_{k}=\sum_{l=0}^{N-1} \Phi_{k, l} d_{l}+w_{k}
$$

where

$$
\Phi_{k, l}=\int_{-\infty}^{\infty} P_{l}(t) P_{k}^{*}(t) d t
$$

and $\left\{w_{k}\right\}$ is additive Gaussian noise with cross correlation [24]

$$
\Omega_{k, l}=E\left[w_{k} w_{l}^{*}\right] \approx 2 N_{0} T \Phi_{k, l} .
$$

Note that since the channel coefficient $\Phi_{k, l}$ is, in general, nonzero for any $k$ and $l$, the noise samples are correlated. This is different from the receivers with an SR branch, where the noise samples are independent.

We can further formulate the discrete channel model (25) in vector form as

$$
\mathbf{y}=\boldsymbol{\Phi} \mathbf{d}+\mathbf{w}
$$

where

$$
\begin{aligned}
\mathbf{d} & =\left[d_{0}, \ldots, d_{N-1}\right]^{\mathrm{T}} \\
\mathbf{y} & =\left[y_{0}, \ldots, y_{N-1}\right]^{\mathrm{T}} \\
\mathbf{w} & =\left[w_{0}, \ldots, w_{N-1}\right]^{\mathrm{T}}
\end{aligned}
$$

and $\Phi$ represents the channel matrix, whose entries are defined by (26). 
With ML detection being computationally infeasible (see [24]), we herein pursue linear solutions based on the standard MMSE detector

$$
\tilde{\mathbf{d}}_{\mathrm{MMSE}}=\operatorname{Dec}\left(\boldsymbol{\Phi}^{\mathrm{H}}\left(\boldsymbol{\Phi} \boldsymbol{\Phi}^{\mathrm{H}}+\boldsymbol{\Omega}\right)^{-1} \mathbf{y}\right)
$$

Since our main focus is on the MR front-end and not on the implementation of detection algorithms, we will use standard matrix inversion to demonstrate the MMSE detection performance. The size of matrix inversion is $N \times N$ in the adopted solution. Clearly, low-complexity linear MMSE detectors, such as those that exploit the banded structure of the channel matrix [26], can be used instead for a more practical implementation.

\section{PRactical CONSIDERATIONS AND RoBUStNess OF THE MR RECEIVER}

So far, we have assumed perfect CSI at the receiver. In practice, however, the channel needs to be estimated, and it is of interest to investigate how the MR schemes perform with the estimated CSI. For this purpose, we adopt two sparse channel estimation schemes, namely, a basis-pursuit (BP) scheme and an orthogonal matching pursuit (OMP) scheme, which are particularly effective for UWA channels [16].

As pointed out in [28], a sparse estimator, be it BP based or OMP based, is subject to a basis mismatch problem when the parameters of the discrete paths are not in the dictionary. For instance, in our case, a path may exist between two nominally allocated dictionary entries. The remaining estimation error is thus determined by the dictionary resolution, i.e., it cannot be eliminated by increasing the signal-to-noise ratio (SNR). Increasing the dictionary size beyond a certain limit is not an option, since the columns of a super-resolution dictionary may be highly correlated, rendering the sparse channel estimation problem illconditioned. Also, unnecessarily high resolution may make the computational complexity too high.

In parallel to other efforts that deal with the basis mismatch problem [29], we propose to estimate the channel using a twostep approach. Using a SISO system as an example, ${ }^{6}$ the first step is to use a standard sparse channel estimation technique (either BP or OMP) to obtain the initial estimates $\left\{\hat{h}_{p}, \hat{\tau}_{p}, \hat{a}_{p}\right\}_{p=0}^{P-1}$ of the channel parameters, where $P$ is the number of identified propagation paths. The second step is to refine the initial estimates by employing a least squares (LS) criterion that aims at compensating for the estimation errors due to the basis mismatch. The resulting estimators are called the phase-compensated BP estimator and the phase-compensated OMP estimator, respectively, because, as will become clear shortly, the major enhancement of these estimators is in the refinement of the initial estimates through effective phase adjustment. Versions without the second step are called the basic BP/OMP estimators.

We start by establishing a cost function to be minimized as the squared distance between the FFT demodulator outputs

\footnotetext{
${ }^{6}$ Extension to multiuser MIMO systems can be pursued by augmenting the
} dictionary to include the propagation paths for all users.

$$
\begin{aligned}
& \mathbf{y}_{\mathrm{FFT}}=\left\{y_{\mathrm{FFT}}(k)\right\}_{k=0}^{N-1}, \text { where } \\
& y_{\mathrm{FFT}}(k)=\int_{0}^{T} v(t) e^{-j 2 \pi k t / T} d t, \quad k=0,1, \ldots, N-1
\end{aligned}
$$

and their predicted values based upon the adjustments of path gains, path delays, and Doppler scaling factors $\hat{\mathbf{y}}_{\mathrm{FFT}}\left(\Delta \hat{h}_{p}, \Delta \hat{\tau}_{p}, \Delta \hat{a}_{p}\right)$, i.e.,

$$
C\left(\Delta \hat{h}_{p}, \Delta \hat{\tau}_{p}, \Delta \hat{a}_{p}\right)=\left\|\mathbf{y}_{\mathrm{FFT}}-\hat{\mathbf{y}}_{\mathrm{FFT}}\left(\Delta \hat{h}_{p}, \Delta \hat{\tau}_{p}, \Delta \hat{a}_{p}\right)\right\|_{(31)}^{2}
$$

To reduce the complexity, we break the optimization problem into two stages: in the first stage, the path gains are fixed and adjustment is only made to the path delays and Doppler scaling factors; in the second stage, adjustment is made only on the path gains. By doing so, we also avoid the ambiguity that exists in joint optimization of $h_{p}, \tau_{p}$, and $a_{p}$.

To proceed, we first give the estimated ICI coefficient [13], $\hat{H}_{p}(k, l)$, obtained from the initial channel estimate, where, defining $\Delta f=1 / T$ and $\beta=(k-l) \Delta f-\hat{a}_{p} f_{l}$, we express

$$
\hat{H}_{p}(k, l)=\hat{h}_{p} e^{-j 2 \pi f_{l} \hat{\tau}_{p}} e^{-j \pi \beta T} \operatorname{sinc}(\pi \beta T) .
$$

Then, we express the updated ICI coefficient (based on the updated channel estimate) $\hat{H}_{p}(k, l)^{\prime}$ as

$$
\begin{aligned}
\hat{H}_{p}(k, l)^{\prime} & =\left(\hat{h}_{p}+\Delta \hat{h}_{p}\right) e^{-j 2 \pi f_{l}\left(\hat{\tau}_{p}+\Delta \hat{\tau}_{p}\right)} e^{-j \pi \beta^{\prime} T} \operatorname{sinc}\left(\pi \beta^{\prime} T\right) \\
& \approx \hat{h}_{p} e^{-j 2 \pi f_{l}\left(\hat{\tau}_{p}+\Delta \hat{\tau}_{p}\right)} e^{-j \pi \beta^{\prime} T} \operatorname{sinc}\left(\pi \beta^{\prime} T\right)
\end{aligned}
$$

where $\beta^{\prime}=(k-l) \Delta f-\left(\hat{a}_{p}+\Delta \hat{a}_{p}\right) f_{l}$. Thus, we can express the predicted FFT output as

$$
\hat{\mathbf{y}}_{\mathrm{FFT}}\left(\Delta \hat{h}_{p}, \Delta \hat{\tau}_{p}, \Delta \hat{a}_{p}\right)=\sum_{p=0}^{P-1} \hat{\mathbf{H}}_{p}^{\prime}\left(\Delta \hat{h}_{p}, \Delta \hat{\tau}_{p}, \Delta \hat{a}_{p}\right) \mathbf{d}
$$

where the entries of the updated channel matrix $\hat{\mathbf{H}}_{p}^{\prime}\left(\Delta \hat{h}_{p}, \Delta \hat{\tau}_{p}, \Delta \hat{a}_{p}\right)$ are specified by (33). Note that since $\Delta \hat{a}_{p} f_{l}$ is very small, (33) can be further simplified as

$$
\begin{aligned}
\hat{H}_{p}(k, l)^{\prime} & \approx \hat{h}_{p} e^{-j 2 \pi f_{l}\left(\hat{\tau}_{p}+\Delta \hat{\tau}_{p}\right)} e^{-j \pi\left(\beta-\Delta \hat{a}_{p} f_{l}\right) T} \operatorname{sinc}(\pi \beta T) \\
& =\underbrace{\hat{h}_{p} e^{-j 2 \pi f_{c} \epsilon_{p}}}_{\hat{h}_{p}^{\prime}} e^{-j 2 \pi \tilde{f}_{l}\left(\hat{\tau}_{p}+\epsilon_{p}\right)} e^{-j \pi \beta T} \operatorname{sinc}(\pi \beta T) \\
& =\tilde{h}_{p}^{\prime} e^{-j 2 \pi \tilde{f}_{l} \epsilon_{p}} \hat{H}_{p}(k, l)
\end{aligned}
$$

where $f_{c}$ is the center subcarrier frequency $\tilde{f}_{l}=f_{l}-f_{c}, \epsilon_{p}=$ $\Delta \hat{\tau}_{p}-\Delta \hat{a}_{p} T / 2$, and $\tilde{h}_{p}=\hat{h}_{p}^{\prime} / \hat{h}_{p}$. By this approximation, the unknown parameters in the first stage of the LS problem are reduced to $\left\{\epsilon_{p}\right\}_{p=0}^{P-1}$. Since the cost function (31) is nonquadratic with respect to $\epsilon_{p}$, we pursue the solution using standard gradient-descent algorithm (GDA), with gradient

$$
\nabla_{p}^{\epsilon}=-2 \operatorname{Re}\left\{\sum_{k=0}^{N-1} e_{k}^{*} \frac{\partial \hat{y}_{\mathrm{FFT}, p}(k)}{\partial \epsilon_{p}}\right\}
$$


where, defining $\hat{y}_{\mathrm{FFT}, p}(k)=\sum_{l=0}^{n-1} \bar{H}_{p}(k, l) d_{l} e^{-j 2 \pi \tilde{f}_{l} \epsilon_{p}}$ with $\bar{H}_{p}(k, l)=\tilde{h}_{p}^{\prime} \hat{H}_{p}(k, l)$

$$
e_{k}=y_{\mathrm{FFT}}(k)-\sum_{p=0}^{P-1} \sum_{l=0}^{N-1} \bar{H}_{p}(k, l) d_{l} e^{-j 2 \pi \tilde{f}_{l} \epsilon_{p}}
$$

is the prediction error of the $k$ th frequency-domain sample $y_{\mathrm{FFT}}(k)$ and

$$
\frac{\partial \hat{y}_{\mathrm{FFT}, p}(k)}{\partial \epsilon_{p}}=\sum_{l=0}^{N-1} \bar{H}_{p}(k, l) d_{l} e^{-j 2 \pi \tilde{f}_{l} \epsilon_{p}}\left(-j 2 \pi \tilde{f}_{l}\right) .
$$

At each iteration of the GDA, the previous value of $\epsilon_{p}(n-1)$ is updated by

$$
\epsilon_{p}(n)=\epsilon_{p}(n-1)-\nabla_{p}^{\epsilon} \cdot \Delta
$$

where $n \geq 1$ is the iteration index, $\Delta$ is a tunable step size, and $\epsilon_{p}(0)=0$. Once $\epsilon_{p}(n)$ converges, we enter the second stage of the LS problem. Note that, with $\epsilon_{p}(n)$ fixed in this stage, the LS solution of $\hat{\mathbf{h}}^{\prime}=\left[\hat{h}_{0}^{\prime} \hat{h}_{1}^{\prime} \ldots \hat{h}_{P-1}^{\prime}\right]^{\mathrm{T}}$ takes the standard form

$$
\hat{\mathbf{h}}^{\prime}=\left(\mathbf{U}^{H} \mathbf{U}\right)^{-1} \mathbf{U}^{H} \mathbf{y}
$$

with $\mathbf{U}=\left[\begin{array}{llll}\mathbf{u}_{0} & \mathbf{u}_{1} & \ldots & \mathbf{u}_{P-1}\end{array}\right]$ and

$$
\mathbf{u}_{p}=\tilde{\mathbf{H}}_{p}(n) \mathbf{d}
$$

where $\left[\tilde{\mathbf{H}}_{p}(n)\right]_{k, l}=\left(\hat{H}_{p}(k, l) / \hat{h}_{p}\right) e^{-j 2 \pi \epsilon_{p} \tilde{f}_{l}}$ is the $(k, l)$ th element of the normalized channel matrix evaluated at the $n$th iteration. We point out that since the initial estimates of the BP or OMP algorithm are refined using this GDA, the requirement for dictionary resolution of the proposed two-step approach can be greatly alleviated, resulting in reduced computational cost and improved robustness of the sparse channel estimators.

\section{NUMERICAL RESULTS}

To demonstrate the effectiveness of the proposed MR designs, we present numerical results comparing their performance with those provided by the conventional, SR solutions. We start with simulated conditions, where perfect CSI is assumed. Then, we give results for the imperfect CSI case, for which the channel estimates are obtained using sparse channel estimators and enhanced versions described in Section V. Finally, we report on the results obtained using experimental data recorded in shallow water $(100 \mathrm{~m})$ in the recent MACE10 and KAM08 experiments.

\section{A. Simulation Results: Known CSI}

1) Results for the User-Specific Doppler Case: The test channels used to simulate a user-specific Doppler case are shown in Fig. 5. The Doppler scaling factors of the two transmitters are set to $-1.0 \times 10^{-3}$ and $1.2 \times 10^{-3}$, which corresponds to relative speeds of $1.5 \mathrm{~m} / \mathrm{s}$ as the transmitter moves away from the receiver, and $1.8 \mathrm{~m} / \mathrm{s}$ as the transmitter moves toward the receiver, respectively. Over this channel, two independent 1024-carrier OFDM signals are transmitted,
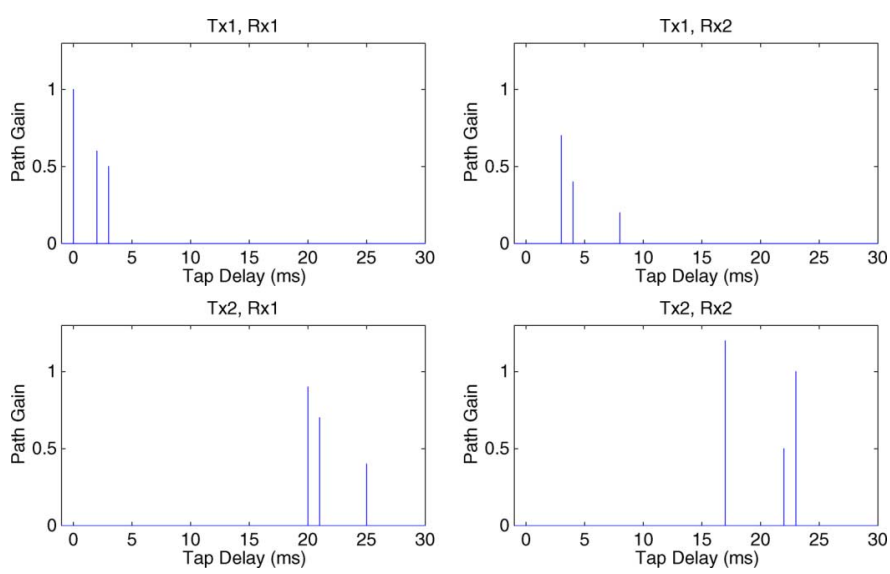

Fig. 5. Multipath profile of the test channel. Doppler rate of the first transmitter is $-1.0 \times 10^{-3}$, and of the second $1.2 \times 10^{-3}$.

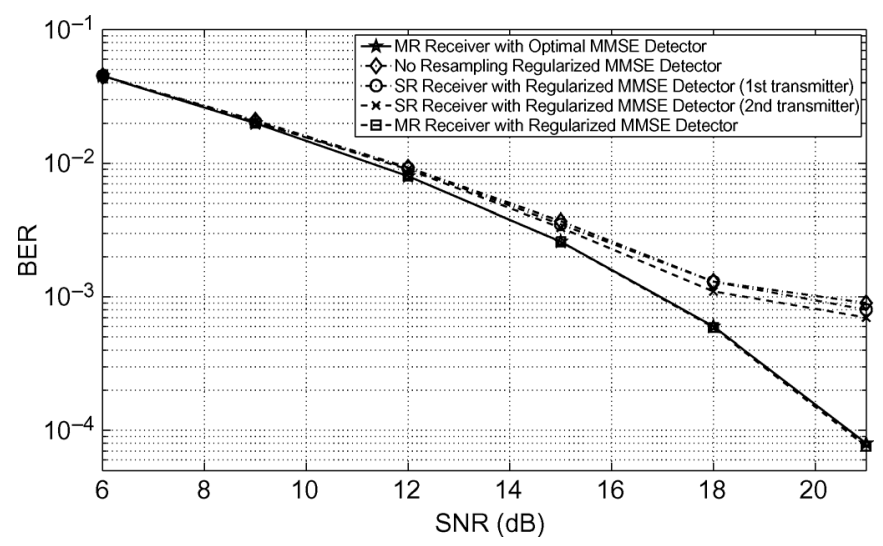

Fig. 6. Performance of linear detection with MR and SR front-ends.

occupying the frequency band from 12 to $20 \mathrm{kHz}$. The intercarrier spacing is $7.8 \mathrm{~Hz}$, which corresponds to an OFDM block duration (excluding the $\mathrm{CP}$ ) of $128 \mathrm{~ms}$. A CP of length $30 \mathrm{~ms}$ is inserted, resulting in a complete OFDM block length of 158 ms.

Fig. 6 shows the results of linear detection, focusing on the performance comparison between the MR and SR front-end solutions. SR includes resampling according to the Doppler scaling factor of the first transmitter and that of the second transmitter. Also included is the case with no resampling. With the transmitters' Doppler scaling factors close in magnitude and opposite in sign, this approach represents the SR receiver [18], whose resampling rate is roughly the average of the two. The results of Fig. 6 pertaining to the regularized linear MMSE detector are obtained with $\epsilon=0.005$ (the average of the nonzero eigenvalues of $\mathbf{H H}^{\mathrm{H}}$ is 0.086 ). Also included are the results for the optimal, SVD-based linear MMSE detector. The regularized MMSE detector with MR obviously performs very close to the optimal solution. More importantly, it offers a substantial performance gain over the SR detectors.

Fig. 7 shows the performance of various detectors proposed for the MR receiver. Included are the regularized MMSE detector, the genie-aided IC detector, in which the interference estimate is obtained using known symbols from the interfering 


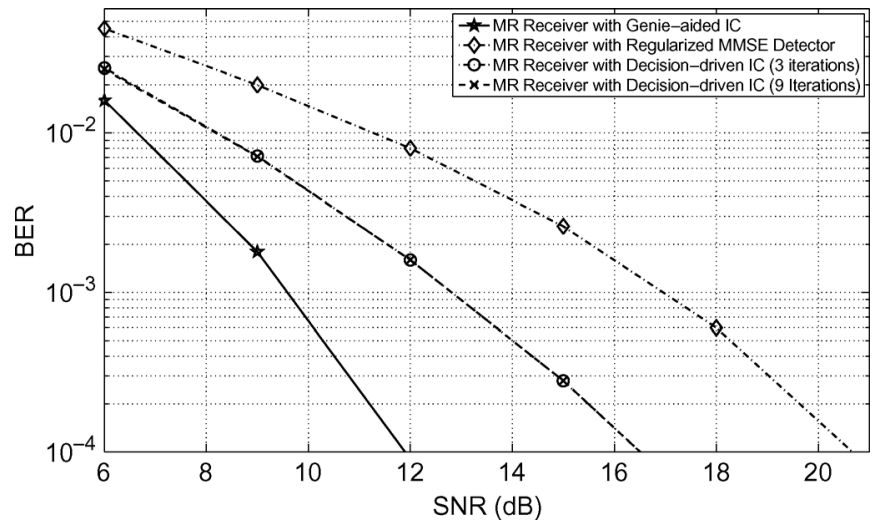

Fig. 7. Performance of MR with linear and nonlinear detection schemes.

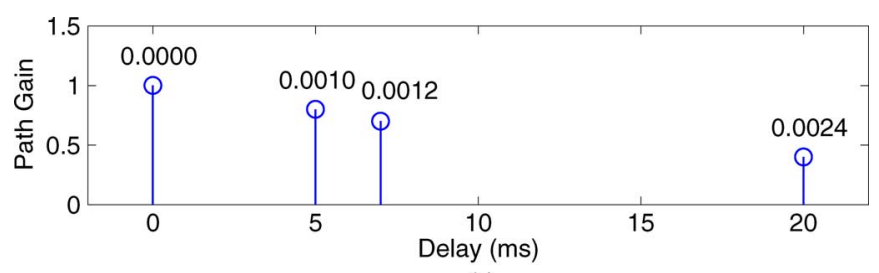

(a)

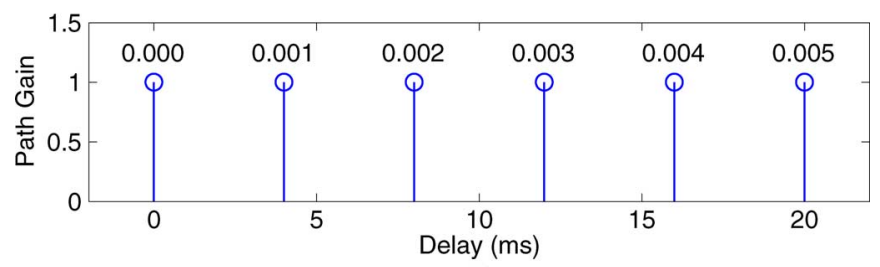

(b)

Fig. 8. Multipath delay profile labeled with path-specific Doppler rates of (a) channel A and (b) channel B.

transmitter, and the decision-driven IC detector. The latter is initialized with decisions from the regularized linear MMSE detector, and employs three or nine iterations. It is observed that the IC detector provides performance that is closer to the genie-aided IC bound, outperforming the regularized MMSE detector by $3 \mathrm{~dB}$ or more. The IC detector takes only three iterations to converge, thus offering a good compromise between performance and complexity. The complexity of ML detection, even in an approximate form, is prohibitive for this test channel (at least 4096 states are required in the Viterbi algorithm).

2) Results for the Path-Specific Doppler Case: Simulation results are reported for the case of a 512-subcarrier OFDM signal transmitted in the frequency band spanning $30-34 \mathrm{kHz}$, i.e., with an intercarrier spacing of $7.8 \mathrm{~Hz}$. We consider two channels, which will be referred to as channel A and channel B, whose features are illustrated in Fig. 8. For both channels, the strongest path is characterized by a zero Doppler rate. Equivalently, we can interpret the Doppler rates shown in Fig. 8 as the residual values after an SR stage matched to the Doppler rate of the strongest path. Hence, no further resampling is needed for the SR demodulator.

First, we compare the magnitude of the ICI coefficients in the equivalent discrete channel model at the output of the demodulator for the SR and MR demodulation cases. Results are visualized in Fig. 9 for channel B referring to the noise-whitened channel model [30]; similar results also hold for channel A. Note

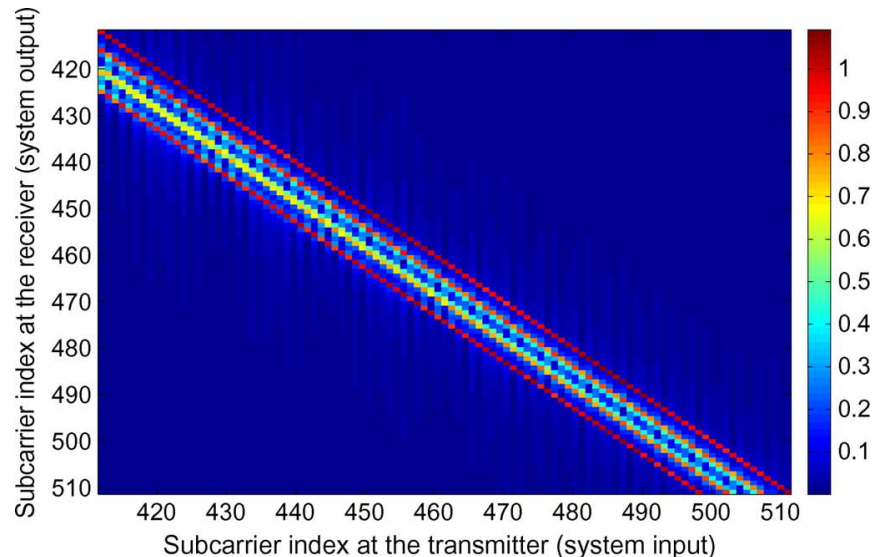

(a)

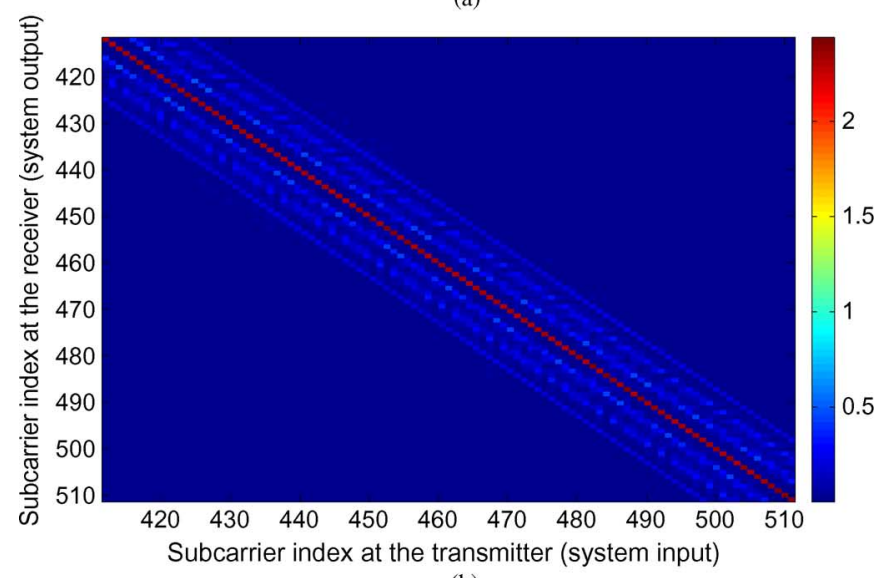

(b)

Fig. 9. Magnitude of the ICI coefficients [see (25) and (26)] obtained for channel B after (a) SR demodulation and (b) MR demodulation.

that the entries on the main diagonal can be interpreted as subcarrier gains, while the other entries are the ICI terms [see (25)]. From Fig. 9, it is clear that the ICI is much stronger for the SR demodulation as compared to the MR demodulation. Namely, the ICI power normalized to the power of the terms on the main diagonal is $+4.5 \mathrm{~dB}$ for the SR demodulation and $-10.7 \mathrm{~dB}$ for the MR demodulation, which corresponds to an ICI-suppression gain of $15.2 \mathrm{~dB}$ provided by the proposed scheme.

Next, we compare the bit error rate (BER) performance of the two systems for uncoded BPSK transmissions. The BERs characterizing the various receivers are shown in Fig. 10 as a function of the SNR, which is defined as the ratio of the signal power and the noise power in $v(t)$. For both demodulation schemes, two different detectors are compared: a symbol-by-symbol detector that neglects all the ICI terms and a linear MMSE-based detector [see (29)]. ML detection is not considered since its complexity is proportional to the number of nonzero ICI terms, which makes it impractical to implement for the set of parameters adopted here. The results demonstrate that the proposed MR demodulation scheme can provide impressive performance gains with respect to the SR demodulation benchmark. Particularly, we notice that for the ICI-ignorant receivers, the BER performance difference is consistent with the ICI-suppression properties discussed earlier. For the ICI-aware receivers, we observe that the BER performance improvement is also significant. In particular, on channel B, the proposed demodulator, combined with a simple symbol-by-symbol detector, offers much 


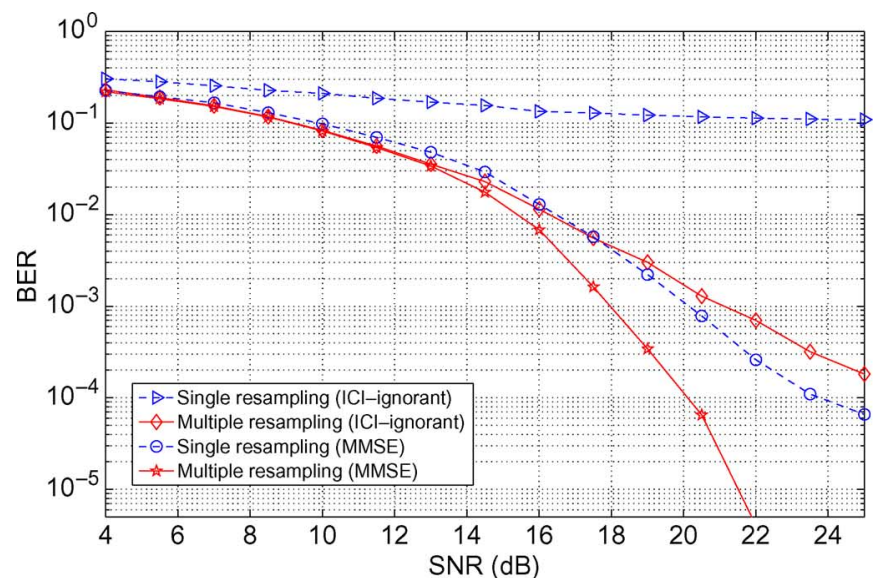

(a)

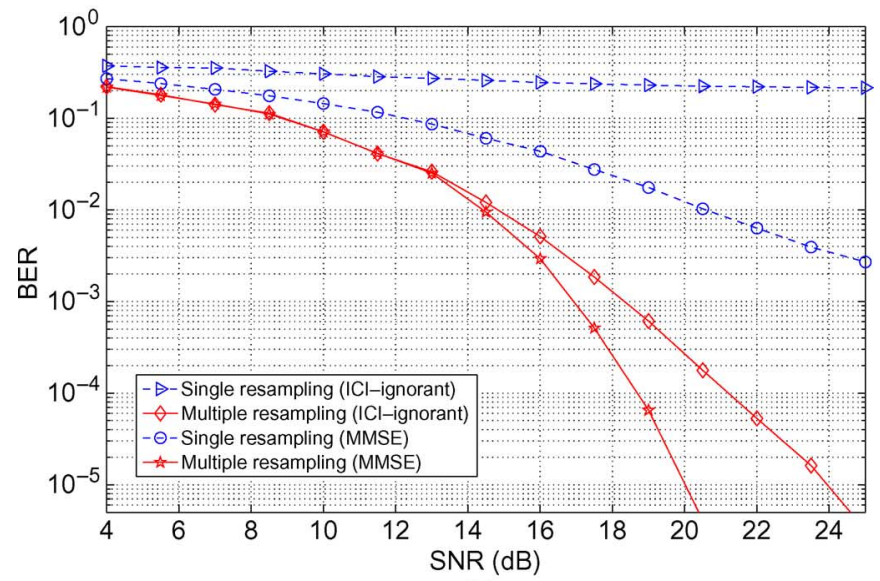

(b)

Fig. 10. BER comparisons between $\mathrm{SR}$ and MR receivers for (a) channel A and (b) channel B.

better BER performance than the standard demodulator combined with a much more complicated linear MMSE detector.

Finally, we compare the performance of the MR and SR receivers over Rician fading channels. We keep the multipath structure as well as the Doppler rates of these channels the same as those of channel A, and randomly generate the gain of each path based on a Rician distribution. The line-of-sight component is selected to be equal to the corresponding path gain of channel A, and the $K$-factor is taken as $12 \mathrm{~dB}$ for all paths (see [31]). In Fig. 11, we present average BER results based on 100 Rician realizations. We observe consistent behavior for the MR and SR receiver performance, which demonstrates that the advantage of MR is preserved in fading conditions.

\section{B. Robustness of MR Detectors}

So far we have demonstrated the BER improvement of the MR solutions when perfect CSI is available at the receiver. We now present results that involve CSI estimation. Specifically, we focus on the two-user cooperative MIMO case in Section VI-A1, where independent data streams are transmitted from nodes subject to user-specific Doppler rates. The multipath structure and Doppler rate for each transmitter/receiver pair are the same as those in Fig. 5. In contrast with the previous section, these channel parameters are not known to the receiver. Instead, they are estimated using the sparse channel

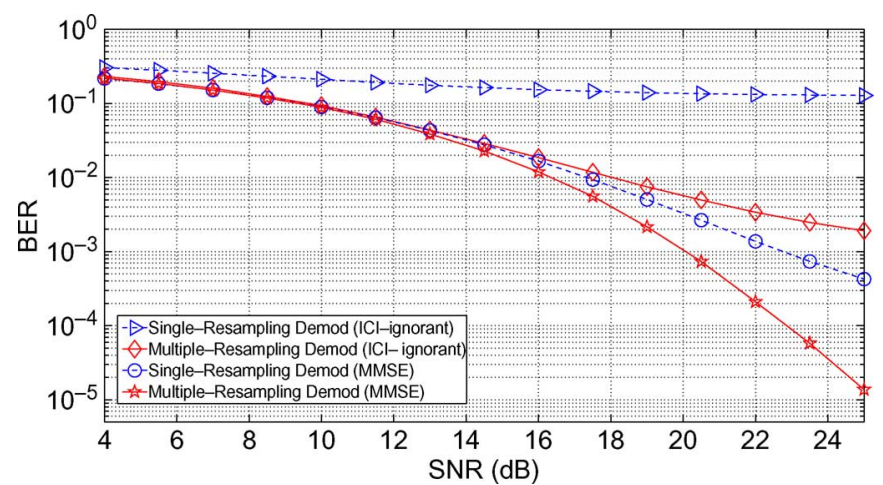

Fig. 11. BER comparisons for the Rician channels (averaged over 100 realizations).

estimation algorithms described in Section V. Here, for simplicity of demonstration, we assume that the channel parameters do not change for multiple consecutive OFDM blocks so that we can devote the first OFDM block for channel estimation and use the resulting channel estimates for decoding of all the following blocks. More practical schemes will be needed as will be discussed in Section VI-C when we deal with results using real experimental data.

With the known transmitted symbols of the first OFDM block, we build a dictionary with a resolution of $62.5 \mu \mathrm{s}$ in the tap delay and $1 \times 10^{-4}$ in the Doppler rate. The dictionary covers a delay spread of $20 \mathrm{~ms}$ and a Doppler rate variation of $\pm 5 \times 10^{-4}$ around the nominal values of each user. To mimic the conditions of a real channel, the path delays of the simulated channels are not constrained to the dictionary values, but are instead uniformly distributed between closest dictionary entries with a zero mean and a range of half of the dictionary resolution.

Fig. 12 shows the actual path locations of the transmitter/receiver pair $(1,1)$ overlapped with the estimated path locations obtained by the BP and OMP algorithms. As expected, due to the basis mismatch problem described in Section $\mathrm{V}$, the number of estimated paths is greater than the actual number of paths for both algorithms, as the sparse channel estimators tend to include several nearby dictionary entries (around the actual one). We observe that the BP-based estimator is subject to more false path locations than the OMP estimator. Consequently, as shown in Fig. 13, the OMP-based MR receiver gives a better BER performance than the BP-based one, for both basic and phase-compensated channel estimation configurations; the former feeds the demodulator/detector with the initial channel estimate, the latter with the updated estimates (39) and (40) (see Section V). From Fig. 13, it is also evident that the performance of the MR receiver with the phase-compensated OMP estimator is uniformly better than that of the basic OMP estimator. The BER performance of such a phase-compensated estimator is actually very close to that of the known CSI case, attesting to this approach's superior ability to compensate for the phase distortions due to basis mismatch. We do not observe such an improvement with the BP estimators, which is due to the inferior quality of the initial channel estimates obtained by the basic BP estimator.

Using the phase-compensated OMP estimator, we now show comparisons of BER performance for the $\mathrm{MR}$ and $\mathrm{SR}$ receivers 


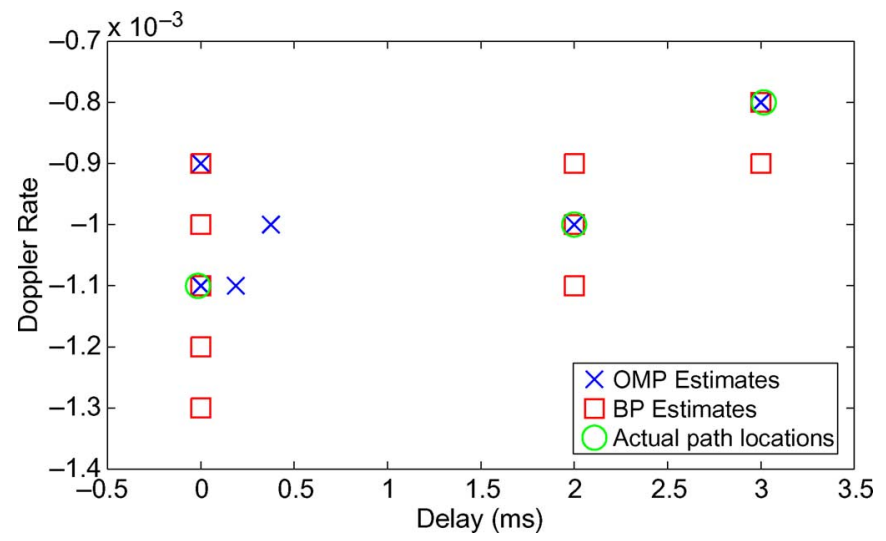

Fig. 12. Path location estimates of the sparse channel estimators.

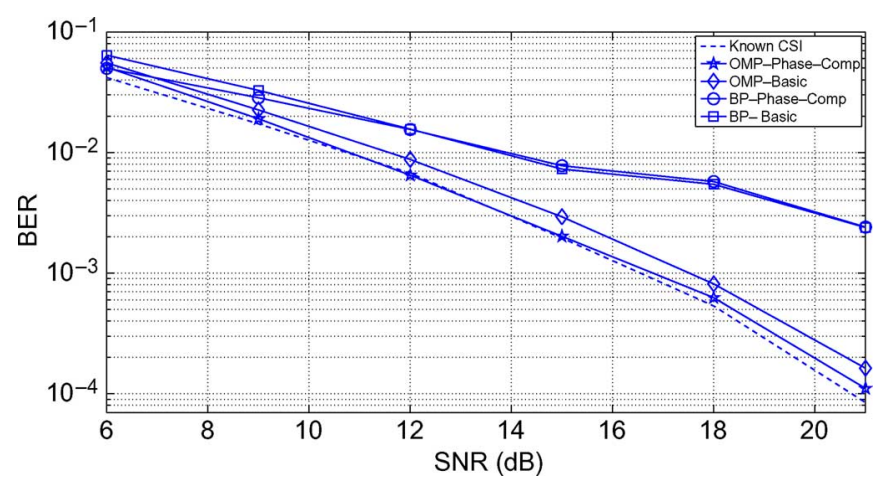

Fig. 13. BER performance comparison between OMP- and BP-based estimators for the test channel. MR receiver with regularized linear MMSE detector is implemented. Basic and phase-compensated (Phase-Comp) receiver configurations correspond to the ones using the initial channel estimate, and the GDA-updated channel estimate (39) and (40), for demodulation and data detection, respectively (see Section V).

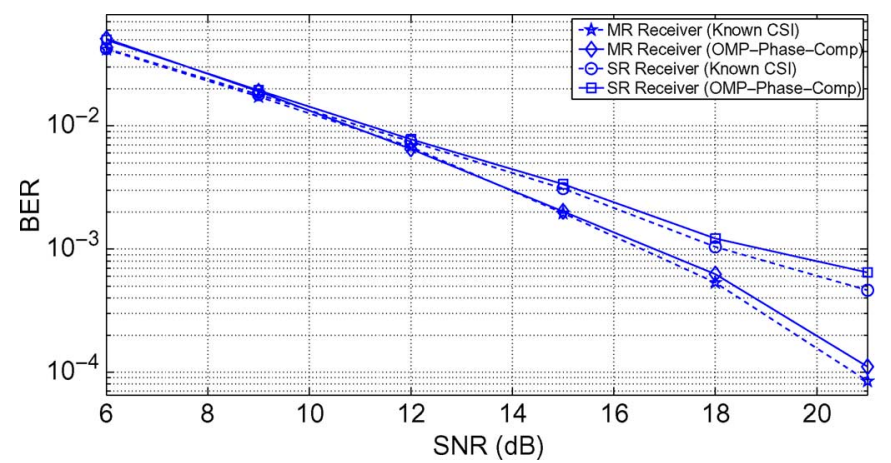

Fig. 14. BER performance comparison between MR and SR receivers (with regularized linear MMSE detector) for the test channel with basis mismatch (path delays not in the dictionary).

with basis mismatch in the path delay. Particularly, as shown in Fig. 14, a sixfold BER reduction is achieved at 21-dB SNR when the MR receiver is employed. The performance difference is very close to that obtained under the perfect CSI conditions.

\section{Experimental Data Results}

To verify the effectiveness of the proposed MR receiver designs with experimental data, we use data recorded in two recent shallow-water (100-m water depth) acoustic communications experiments, namely, the Mobile Acoustic Communications Experiment (MACE10) conducted in June 2010 off the southeastern coast of Massachusetts, and the Kauai Acomms MURI (KAM08) experiment conducted in June 2008 off the western coast of Kauai, HI.

1) Results Obtained With the MACE10 Data: During the MACE10 experiment, one mobile source, towed at a nominal speed of about $1 \mathrm{~m} / \mathrm{s}$, and two fixed receivers were used. The source was equipped with four International Transducer Corporation (ITC) 1007 spherical transducers, submerged at a depth of between 30 and $60 \mathrm{~m}$. The transducer spacings were $48 \mathrm{~cm}$ between the first and second ones, $42 \mathrm{~cm}$ between the second and third ones, and $48 \mathrm{~cm}$ between the last two. The two receivers, both with four receiving elements, were suspended from small surface buoys. The interelement spacing and sampling rate were $20 \mathrm{~cm}$ and $50 \mathrm{kHz}$, respectively, for both. We particularly focus on data recorded when the source was about 1.3 and $4.3 \mathrm{~km}$ from the two receivers. The corresponding transmitted signals contained 15 blocks of 512-subcarrier CP-OFDM signals employing QPSK modulation and 16-ms cyclic prefix. The sampling rate before digital-to-analog conversion (DAC) was $F_{s}=$ $10^{7} / 256=39.0625 \mathrm{kHz}$, and the bandwidth was $B=F_{s} / 8 \approx$ $5 \mathrm{kHz}$, resulting in a subcarrier spacing of about $10 \mathrm{~Hz}$. The lowest frequency subcarrier was located at $f_{0}=10.580 \mathrm{kHz}$. Interested readers are referred to [31] for examples of measured impulse response on real UWA channels.

The existing experimental configuration supports transmission from a single source to multiple receivers. To mimic the conditions for multiuser transmissions, where independent streams are emitted from multiple spatially separated nodes, we use received signals that correspond to two consecutive blocks recorded at the two spatially separated receivers and sum them to form a superimposed signal - effectively each block of the superimposed signal corresponds to 2048 transmitted bits. The superimposed signal thus contains two independent data streams with independent multipath structures and different nominal Doppler rates. The latter is due to different relative speeds between the transmitters and the receiver. Since the difference between the relative speeds is small, the difference between the nominal Doppler rates is also small-on the order of $5 \times 10^{-4}$. To mimic scenarios with larger nominal Doppler rate differences, we resample the received signals from different receivers at different rates before summing them. Effectively, we introduce an additional Doppler scaling to the received signal, and as a result, we are able to control the nominal Doppler rate difference in the received signal. As an example, we generate superimposed signals with $4.4 \times 10^{-3}$ Doppler rate differences - the Doppler rates for the two users are the same in magnitude but opposite in sign. The equivalent speed difference between the two paths is $6.6 \mathrm{~m} / \mathrm{s}$.

We implement the SR and MR receiver designs with the OMP-based channel estimator, as discussed in Section VI-B. Since the average nominal Doppler rate of the two users is zero, the optimal SR receiver performs no resampling. For the MR receiver, two MR branches suffice as the path-specific Doppler rate difference is on the order of $2 \times 10^{-4}$ for each user. Therefore, we adopt a simplified MR receiver implementation with an SR branch for each user. The resampling rate is set according to the nominal Doppler rate of that user. The receivers are implemented in the decision-directed fashion. 


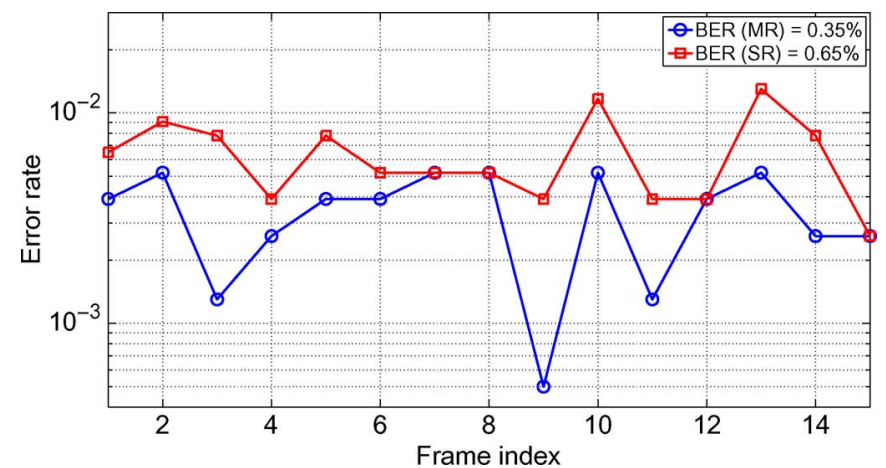

Fig. 15. MACE10 BER performance comparison between $\mathrm{MR}$ and SR receivers in decision-directed mode $\left(\Delta a=4.4 \times 10^{-3}\right)$.

That is, we start the data detection from channel estimates obtained with pilots signals - the pilot assignment is similar to that in [16]; then, we use the channel estimates to perform regularized MMSE detection (Section III-B1), whose tentative decisions are finally used for IC detection (Section III-B2). The detected symbols are then used together with the pilot signals for the next round of iterative channel estimation and detection.

In Fig. 15, we show the BER performance comparison between SR and MR receivers with $4.4 \times 10^{-3}$ Doppler rate difference, where the BER results refer to those obtained at the fifth iteration in a $2 \times 4$ system configuration. On average, the MR receiver results in a twofold BER reduction over the SR receiver. The BER reduction improves with the difference in Doppler. We point out that using detected symbols for channel estimation in decision-directed mode may subject the overall system to performance degradation due to error propagation. A solution is to involve explicit channel coding in the loop where decoded symbols are fed back for channel estimation. As a preliminary study, we consider a coded system with each 512-subcarrier QPSKmodulated OFDM block spanning two length 512, rate 0.9 lowdensity parity-check (LDPC) codewords. The channel decoder takes soft-information, i.e., log-likelihood ratios (LLRs), generated by the IC detector as its input, and outputs updated LLRs (after ten decoding iterations) that are used to obtain coded bit decisions. The decoded bits are then remapped to modulation symbols, which are exploited in the next iteration for channel estimation, detection, and LDPC decoding. In Fig. 16, we show the BER comparisons of MR and SR receivers of the above coded system. We observe a threefold uncoded BER reduction for the $8.8 \times 10^{-3}$ Doppler rate difference after three iterations of channel estimation, detection, and decoding-for both receivers, the coded BERs drop to zero since they are too low to be captured by the limited number of transmissions. The performance gain of the MR over the SR receiver increases as a result of the improved channel estimation quality when modulation symbols mapped from the decoded bits are used for channel estimation.

2) Results Obtained With the KAM08 Data: Last, we consider communication data collected in the KAM08 experiment. We focus on the results for a 512-carrier OFDM system, where BPSK modulation was used. The signal spanned a frequency band between 12 and $20 \mathrm{kHz}$ and had a cyclic prefix of $20 \mathrm{~ms}$, which implied a block duration of $276 \mathrm{~ms}$ including the $\mathrm{CP}$. The experimental data were collected while the transmitter was

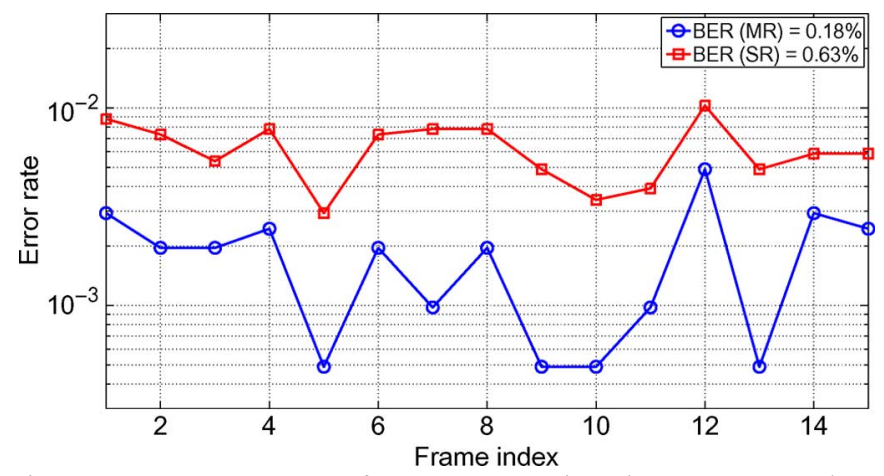

Fig. 16. MACE10 BER performance comparison between $\mathrm{MR}$ and SR receivers with channel coding $\left(\Delta a=8.8 \times 10^{-3}\right)$

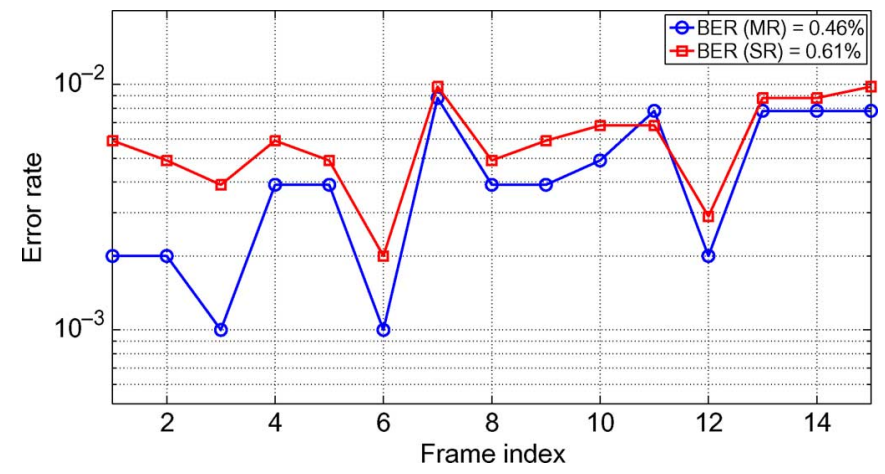

Fig. 17. KAM08 BER performance comparison between MR and SR receivers with channel coding $\left(\Delta a=4.4 \times 10^{-3}\right)$.

moving. The transmitter was submerged at a depth spanning 20-50 m, depending on the specific experiment, and was towed at a nominal speed of $3 \mathrm{kn}$ (i.e., about $1.54 \mathrm{~m} / \mathrm{s}$ ). The receiver had a 16-element vertical array. The sampling rate at the receiver was $50 \mathrm{kHz}$. The interelement spacing was $3.75 \mathrm{~m}$, with the top element deployed at a nominal depth of $42.25 \mathrm{~m}$. Particularly, we consider the case when the transmitter/receiver separation was approximately $2 \mathrm{~km}$, and the towing ship was moving toward the fixed receiver, with the transmitting transducer being about $25 \mathrm{~m}$ below the sea surface.

Adopting the same approach as described in Section VI-C1, we predistort the received signals to form composite signals that have controlled Doppler rate differences. We notice that, compared to the MACE10 experiment, the received signals are subject to lower SNR and, therefore, lead to an inferior channel estimation quality. To make up for the performance loss, we use $2 \times 6$ systems instead of $2 \times 4$ systems in Section VI-C1. We see a similar trend of the MR receivers to perform better than the SR receivers, as shown in Fig. 17. However, attributed to the inaccurate channel estimates, the performance advantages of the MR receivers are not as pronounced.

\section{SUMMARY AND CONCLUSION}

We have investigated the problem of multiuser detection when different users' signals are subject to different Doppler distortions. This problem is also mathematically analogous to the problem of path-specific Doppler. Doppler-compensated receiver front-end designs are presented. In particular, we focused on OFDM transmissions in the context of multiuser MIMO and single-user SISO scenarios. In the former case, a centralized receiver was considered (with collocated receiver 
elements) communicating with multiple distributed users, which transmit independent data streams simultaneously in the same frequency band. We pointed out the inadequacy of standard SR designs and proposed a set of new designs based on MR front-ends. For multiuser MIMO systems, each branch corresponds to the Doppler rate of a particular user, provided that path-specific Doppler for each user can be neglected, whereas for single-user SISO systems, a resampling branch is needed for each cluster of arrivals that share a common Doppler rate. The new designs have the advantage of compensating for the user- and/or path-specific Doppler distortions, therefore avoiding strong interuser and/or intercarrier inference inherent to SR designs. Via extensive simulations and experimental data studies, we have demonstrated that compensation of userand/or path-specific Doppler translates into performance gains in terms of BER improvement and ICI power reduction.

\section{REFERENCES}

[1] Y. Emre, V. Kandasamy, T. M. Duman, P. Hursky, and S. Roy, "Multiinput multi-output OFDM for shallow-water UWA communications," in Proc. Acoust. Conf., Jun. 2008, pp. 13-17.

[2] K. Tu, D. Fertonani, T. M. Duman, and P. Hursky, "Mitigation of intercarrier interference in OFDM systems over underwater acoustic channels," in Proc. IEEE OCEANS Conf., Bremen, Germany, May 2009, DOI: 10.1109/OCEANSE.2009.5278270.

[3] B. Li, J. Huang, S. Zhou, K. Ball, M. Stojanovic, L. Freitag, and P. Willett, "MIMO-OFDM for high rate underwater acoustic communications," IEEE J. Ocean. Eng., vol. 34, no. 4, pp. 634-645, Oct. 2009.

[4] M. Stojanovic, "MIMO OFDM over underwater acoustic channels," in Proc. 43rd Asilomar Conf. Signals Syst. Comput., Pacific Grove, CA, Nov. 2009, pp. 605-609.

[5] D. Gesbert, M. Kountouris, R. W. Heath, C. Chae, and T. Sälzer, "From single user to multiuser communications: Shifting the MIMO paradigm," IEEE Signal Process. Mag., vol. 24, no. 5, pp. 36-46, Oct. 2007.

[6] W. Yu and W. Rhee, "Degrees of freedom in wireless multiuser spatial multiplex systems with multiple antennas," IEEE Trans. Commun., vol. 54, no. 10, pp. 1747-1753, Oct. 2006.

[7] M. Vayapeyam, S. Vedentam, U. Mitra, J. Preisig, and M. Stojanovic, "Distributed space-time cooperative schemes for underwater acoustic communications," IEEE J. Ocean. Eng., vol. 33, no. 4, pp. 489-501, Oct. 2008

[8] Q. H. Spencer, A. L. Swindlehurst, and M. Haardt, "Zero-forcing methods for downlink spatial multiplexing in multiuser MIMO channels," IEEE Trans. Signal Process., vol. 52, no. 2, pp. 461-471, Feb. 2004.

[9] N. H. Dawod, R. Hafez, and I. Marsland, "Uplink multiuser MIMOOFDM in widely spaced antenna arrays," in Proc. Can. Conf. Electr. Comput. Eng., May 2005, pp. 155-159.

[10] S. Venkatesan, A. Lozano, and R. Valenzuela, "Network MIMO: Overcoming intercell interference in indoor wireless systems," in Proc. 41st Asilomar Conf. Signals Syst. Comput., Pacific Grove, CA, Nov. 2007, pp. 83-87.

[11] L. Zou, Q. Chang, C. Xiu, and Q. Zhang, "Channel estimation and ICI cancellation for OFDM systems in fast time-varying environments," IEICE Trans. Commun., vol. E91-B, no. 4, pp. 1203-1206, Apr. 2008.

[12] X. Huang and $\mathrm{H}$. Wu, "Robust and efficient intercarrier interference mitigation for OFDM systems in time-varying fading channels," IEEE Trans. Veh. Technol., vol. 56, no. 5, pp. 2517-2528, Sep. 2007.

[13] S. Mason, C. Berger, S. Zhou, K. Ball, L. Freitag, and P. Willett, "An OFDM design for underwater acoustic channels with Doppler spread," in Proc. Digital Signal Process. Workshop/5th IEEE Signal Process. Edu. Workshop, Jan. 2009, pp. 138-143.

[14] S. J. Hwang and P. Schniter, "Efficient multicarrier communication for highly spread underwater acoustic channels," IEEE J. Sel. Areas Commun., vol. 26, no. 9, pp. 1674-1683, Dec. 2008.

[15] B. Li, S. Zhou, M. Stojanovic, L. Freitag, and P. Willett, "Multicarrier communication over underwater acoustic channels with nonuniform Doppler shifts," IEEE J. Ocean. Eng., vol. 33, no. 2, pp. 198-209, Apr. 2008.
[16] C. R. Berger, S. Zhou, J. Preisig, and P. Willett, "Sparse channel estimation for multicarrier underwater acoustic communication: From subspace methods to compressed sensing," IEEE Trans. Signal Process., vol. 58, no. 3, pp. 1708-1721, Mar. 2010.

[17] W. Li and J. C. Presig, "Estimation of rapidly time-varying sparse channels," IEEE J. Ocean. Eng., vol. 32, no. 4, pp. 927-939, Oct. 2007.

[18] S. Yerramalli and U. Mitra, "On optimal resampling for OFDM signaling in doubly-selective underwater acoustic channels," in Proc. IEEE OCEANS Conf., Quebec City, QC, Canada, Sep. 2008, DOI: 10.1109/OCEANS.2008.5152064

[19] K. Davidson, "MACE10-Mobile Acoustic Communications Experiment 2010-Quick-Look Cruise Report,” Tech. Rep., Aug. 2010.

[20] W. S. Hodgkiss, H. C. Song, M. Badiey, A. Song, and M. Siderius, "Kauai Acomms MURI 2008 (KAM08) Experiment-Trip Report," Tech. Rep., Jul. 2008.

[21] A. R. S. Bahai, B. R. Saltzberg, and M. Ergen, Multi-Carrier Digital Communications: Theory and Applications of OFDM. New York: Kluwer, 1999, ch. 2.

[22] T. M. Duman and A. Ghrayeb, Coding for MIMO Communication Systems. New York: Wiley, 2007, ch. 3.

[23] Z.-H. Wang, S. Zhou, G. B. Giannakis, C. R. Berger, and J. Huang, "Frequency-domain oversampling for zero-padded OFDM in underwater acoustic communications," in Proc. IEEE Global Commun. Conf., Miami, FL, Dec. 2010, DOI: 10.1109/GLOCOM.2010. 5683290.

[24] K. Tu, "Multi-carrier communications over underwater acoustic channels," Ph.D. dissertation, Schl. Electr. Comput. Energy Eng., Arizona State Univ., Tempe, AZ, Dec. 2011.

[25] G. H. Golub and C. F. V. Loan, Matrix Computations, 3rd ed. Baltimore, MD: Johns Hopkins Univ. Press, 1999.

[26] L. Rugini, P. Banelli, and G. Leus, "Low-complexity banded equalizers for OFDM systems in Doppler spread channels," EURASIP J. Appl. Signal Process., 2006, Article ID 67404.

[27] A. F. Molisch, M. Toeltsch, and S. Vermani, "Iterative methods for cancellation of intercarrier interference in OFDM systems," IEEE Trans. Veh. Technol., vol. 56, no. 4, pp. 2158-2167, Jul. 2007.

[28] Y. Chi, L. L. Scharf, A. Pezeshki, and A. R. Calderbank, "Sensitivity to basis mismatch in compressed sensing," IEEE Trans. Signal Process., vol. 59, no. 5, pp. 2182-2195, May 2011.

[29] T. Huang, Y. Liu, H. Meng, and X. Wang, "Adaptive matching pursuit with constrained total least squares," EURASIP J. Adv. Signal Process., vol. 2012, no. 1, pp. 76-87, Apr. 2012.

[30] G. D. Forney, Jr., "Maximum-likelihood sequence estimation of digital sequences in the presence of intersymbol interference," IEEE Trans. Inf. Theory, vol. IT-18, no. 3, pp. 363-379, May 1972.

[31] A. Radosevic, J. G. Proakis, and M. Stojanovic, "Statistical characterization and capacity of shallow water acoustic channels," in Proc. IEEE OCEANS Conf., Bremen, Germany, May 2009, DOI: 10.1109/ OCEANSE.2009.5278349.

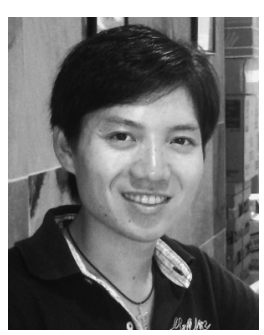

Kai Tu received the B.S. degree (with honors) in electrical engineering from the East China Normal University, Shanghai, China, in 1999 and the M.S. and $\mathrm{Ph} . \mathrm{D}$. degrees in electrical engineering from the Arizona State University (ASU), Tempe, in 2008 and 2011.

From 1999 to 2004, he was a Consultant and Application Engineer at the Shanghai Representative Office of Nicolet Instrument Technologies, Inc., Madison, WI. In August 2004, he was admitted to the M.S. program of the Department of Electrical Engineering at ASU, where he conducted research on speech codec, adaptive beamforming and sound localization algorithms. In August 2005, he transferred to the Arts, Media and Engineering program of the same department, to work on particle filtering for sound location, and probabilistic models in the context of mediated active learning. In January 2008, he joined the Wireless Communications Lab at ASU, and was admitted to the Ph.D. program in August 2008. His research interest is in adaptive equalization, interference cancellation, Doppler mitigation, and capacity analysis of time-varying multipath channels, mostly in the context of MIMO-OFDM underwater acoustic communications. Currently, he works at Qualcomm Inc., San Diego, CA. 


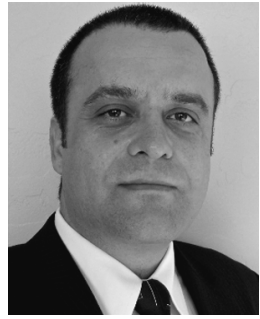

Tolga M. Duman (S'95-M'98-SM'03-F'11) received the B.S. degree from Bilkent University, Bilkent, Ankara, Turkey, in 1993 and the M.S. and $\mathrm{Ph} . \mathrm{D}$. degrees from Northeastern University, Boston, MA, in 1995 and 1998, respectively, all in electrical engineering.

$\mathrm{He}$ is a Professor of Electrical and Electronics Engineering Department of Bilkent University, and is on leave from the School of Electrical, Computer and Energy Engineering, Arizona State University, Tempe. Before joining Bilkent University in August 2012, he was with the Electrical Engineering Department, Arizona State University, first as an Assistant Professor (1998-2004), then as an Associate Professor (2004-2008), and starting August 2008 as a Professor. His publications include a book Coding for MIMO Communication Systems (New York, NY: Wiley, 2007), over 50 journal papers, and over 100 conference papers. His current research interests are in systems, with particular focus on communication and signal processing, including wireless and mobile communications, coding/modulation, coding for wireless communications, data storage systems, and underwater acoustic communications.

Dr. Duman is a recipient of the National Science Foundation CAREER Award and the IEEE Third Millennium medal. He served as an editor for the IEEE TRANSACTIONS ON WIRELESS COMMUNICATIONS (2003-2008), the IEEE TRANSACTIONS ON COMMUNICATIONS (2007-2012), and the IEEE ONLINE JOURNAL OF SURVEYS AND TUTORIALS (2002-2007). He is currently the coding and communication theory area editor for the IEEE TRANSACTIONS ON COMMUNiCATIONS (2011-present) and an editor for Elsevier's Physical Communications Journal (2010-present).

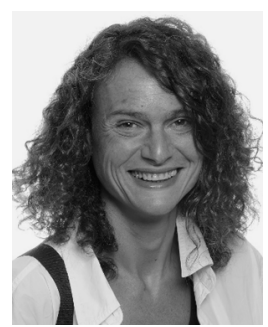

Milica Stojanovic (S'08-F'10) graduated from the University of Belgrade, Belgrade, Serbia, in 1988 and received the M.S. and Ph.D. degrees in electrical engineering from Northeastern University, Boston, MA, in 1991 and 1993.

After a number of years with the Massachusetts Institute of Technology (MIT), Cambridge, where she was a Principal Scientist, she joined the faculty of Electrical and Computer Engineering Department, Northeastern University, in 2008. She is also a Guest Investigator at the Woods Hole Oceanographic Institution, Woods Hole, MA, and a Visiting Scientist at MIT. Her research interests include digital communications theory, statistical signal processing and wireless networks, and their applications to underwater acoustic communication systems.

Dr. Stojanovic is an Associate Editor for the IEEE Journal OF OCEANIC ENGINEERING and the IEEE TRANSACTIONS ON SignAL PROCESSING.

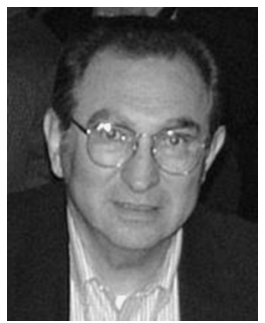

John G. Proakis (S'58-M'62-F'84-LF'99) received the B.S.E.E. degree from the University of Cincinnati, Cincinnati, OH, in 1959, the M.S.E.E. degree from the Massachusetts Institute of Technology (MIT), Cambridge, in 1961, and the Ph.D. degree from Harvard University, Cambridge, MA, in 1967.

$\mathrm{He}$ is an Adjunct Professor at the University of California San Diego, La Jolla, and a Professor Emeritus at Northeastern University, Boston, MA He was a faculty member at Northeastern University from 1969 through 1998 and held the following academic positions: Associate Professor of Electrical Engineering (1969-1976), Professor of Electrical Engineering (1976-1998), Associate Dean of the College of Engineering and Director of the Graduate School of Engineering (1982-1984), Interim Dean of the College of Engineering (1992-1993), and Chairman of the Department of Electrical and Computer Engineering (1984-1997). Before joining Northeastern University, he worked at GTE Laboratories and the MIT Lincoln Laboratory. His professional experience and interests are in the general areas of digital communications and digital signal processing. He is the author of the book Digital Communications (New York, NY: McGraw-Hill, 2001, 4th edition), and coauthor of the books Introduction to Digital Signal Processing (Upper Saddle River, NJ: Prentice-Hall, 2007, 4th edition), Digital Signal Processing Laboratory (Englewood Cliffs, NJ: Prentice-Hall, 1991), Advanced Digital Signal Processing (New York, NY: Macmillan, 1992), Algorithms for Statistical Signal Processing (Upper Saddle River, NJ: Prentice-Hall, 2002), Discrete-Time Processing of Speech Signals (New York, NY: Macmillan, 1992, IEEE Press, 2000), Communication Systems Engineering (Upper Saddle River, NJ: Prentice-Hall, 2002, 2nd edition), Digital Signal Processing Using MATLAB V.4 (Boston, MA: Brooks/Cole-Thomson Learning, 2007, 2nd edition), Contemporary Communication Systems Using MATLAB (Boston, MA: Brooks/Cole-Thomson Learning, 2004, 2nd edition), and Fundamentals of Communication Systems (Upper Saddle River, NJ: Prentice-Hall, 2005). 